\title{
Developmental organization of central neurons in the adult Drosophila ventral nervous system
}

Shepherd, David; Sahota, Virender; Court, Robert; Williams, Darren W.; Truman, James

\section{Journal of Comparative Neurology}

DOI:

$10.1002 /$ cne. 24690

Published: 15/10/2019

Peer reviewed version

Cyswllt i'r cyhoeddiad / Link to publication

Dyfyniad o'r fersiwn a gyhoeddwyd / Citation for published version (APA):

Shepherd, D., Sahota, V., Court, R., Williams, D. W., \& Truman, J. (2019). Developmental organization of central neurons in the adult Drosophila ventral nervous system. Journal of Comparative Neurology, 527(15), 2573-2598. https://doi.org/10.1002/cne.24690

\footnotetext{
Hawliau Cyffredinol / General rights

Copyright and moral rights for the publications made accessible in the public portal are retained by the authors and/or other copyright owners and it is a condition of accessing publications that users recognise and abide by the legal requirements associated with these rights.

- Users may download and print one copy of any publication from the public portal for the purpose of private study or research.

- You may not further distribute the material or use it for any profit-making activity or commercial gain

- You may freely distribute the URL identifying the publication in the public portal ?
}

Take down policy

If you believe that this document breaches copyright please contact us providing details, and we will remove access to the work immediately and investigate your claim. 


\section{Developmental Organisation of Central Neurons in the Adult Drosophila Ventral Nervous System}

\begin{tabular}{|c|c|}
\hline Journal: & Journal of Comparative Neurology \\
\hline Manuscript ID & JCN-18-0278.R2 \\
\hline Wiley - Manuscript type: & Research Article \\
\hline Keywords: & $\begin{array}{l}\text { Neurogenesis, Neuropil, Development, Neuroblast, Lineage, Hemilineage, } \\
\text { MARCM, RRID:AB_2337254, RRID:AB_221569, RRID:AB_528121, } \\
\text { RRID:AB_528402, RRID:AB_2341099,RRID:AB_2340855, } \\
\text { RRID:AB_2340694, RRID:SCR_001935, RRID:SCR_002609, } \\
\text { RRID:SCR_014199, RRID:SCR_004229, RRID:SCR_002234 }\end{array}$ \\
\hline
\end{tabular}

SCHOLARONE $^{\text {m }}$
Manuscripts $^{\text {Manusion }}$ 


\section{Developmental Organisation of Central Neurons in the Adult Drosophila Ventral Nervous System}

Short title: Neuronal lineages in the Drosophila

David Shepherd $^{1 *}$, Virender Sahota ${ }^{1}$, Robert Court ${ }^{2}$, Darren W. Williams ${ }^{3}$ and James W. Truman $^{4,5}$

1 School of Natural Sciences, Bangor University, Bangor, Gwynedd, LL57 2UW, UK 2 School of Informatics, University of Edinburgh, Edinburgh, UK 3 Centre for Developmental Neurobiology, King's College London, London SE1 1UL UK 4 Howard Hughes Medical Institute, Janelia Research Campus, Ashburn, VA, USA 5 Friday Harbor Laboratories, University of Washington, Friday Harbor, WA 98250 USA

* Correspondence: David Shepherd, School of Natural Sciences, Environment Centre Wales, Deiniol Rd, Bangor University, Bangor, Gwynedd, LL57 2UW, UK 


\section{Acknowledgments}

The authors are grateful for the support of the Howard Hughes Medical Institute (HHMI) Janelia Research Campus. DS was funded by Higher Education Funding Council for Wales and the HHMI visiting scientist program. VS was funded by Bangor University. DWW was funded by the HHMI visiting scientist program. JWT was funded by HHMI. We also thank Todd Laverty and the FlyCore staff at Janelia for help with fly crosses, Alison Howard for administrative help and Haluk Lacin for sharing his unpublished work. 


\begin{abstract}
We have used MARCM to reveal the adult morphology of the post embryonically produced neurons in the thoracic neuromeres of the Drosophila VNS. The work builds on previous studies of the origins of the adult VNS neurons (Harris et al., 2015; Shepherd et al., 2016; Truman et al., 2004) to describe the clonal organization of the adult VNS. We present data for 58 of 66 postembryonic thoracic lineages, excluding the motor neuron producing lineages (15 and 24) which have been described elsewhere (Baek \& Mann, 2009; Brierley et al., 2012). MARCM labels entire lineages but where both $A$ and $B$ hemilineages survive [e.g. lineages $19,12,13,6,1,3,8,11$ ], the two hemilineages can be discriminated and we have described each hemilineage separately. Hemilineage morphology is described in relation to the known functional domains of the VNS neuropil and based on the anatomy we are able to assign broad functional roles for each hemilineage. The data show that in a thoracic hemineuromere, 16 hemilineages are primarily involved in controlling leg movements and walking, 9 are involved in the control of wing movements, and 10 interface between both leg and wing control. The data provide a baseline of understanding of the functional organization of the adult Drosophila VNS. By understanding the morphological organization of these neurons, we can begin to define and test the rules by which neuronal circuits are assembled during development and understand the functional logic and evolution of neuronal networks.
\end{abstract}

Key Words: Neurogenesis, Neuropil, Anatomy, Development, Neuroblast, Hemilineage, Lineage, MARCM, RRID:AB_2337254, RRID:AB_221569, RRID:AB_528121, RRID:AB_528402, RRID:AB_2341099, RRID:AB_2340855, RRID:AB_2340694, RRID:SCR_001935, RRID:SCR_002609, RRID:SCR_014199, RRID:SCR_004229, RRID:SCR_002234 


\section{Background}

Understanding how the nervous system is able integrate sensory inputs to generate and control motor outputs remains a central challenge of neuroscience. Although the massive cellular complexity of the central nervous system (CNS) presents challenges, there are approaches that make analysis possible. Within most nervous systems it is possible to unpack the complexity by recognizing that most neurons can be placed into identifiable units based on their morphological and physiological characteristics. These elements can then be placed into functional networks that can go some way to explaining how they generate the outputs to drive behavior. This approach can be best seen in work on the vertebrate brainstem and spinal cord. In the spinal cord neurons can be placed into discrete populations of interneurons, which share clearly defined roles in the formation of functional neural circuits (Grillner \& Jessell, 2009). More remarkably the functional populations of interneurons are developmentally based being generated by specific, identified neuronal progenitor cells (Grillner \& Jessell, 2009). The finding that specific stem cells generate pools of functionally similar interneurons in the vertebrate spinal cord appears to be conserved with the progenitor cells and transcription factors that define different interneuron classes conserved from lower vertebrates to mammals (Lupo, Harris, \& Lewis, 2006). The clear implication of this work is that, at least for locomotor control circuitry, these developmental units are also likely to be functional units related serving similar roles in the control circuitry.

The same organizational principles of distinct neuronal classes are also found in the insect ventral nervous system (VNS), which, like the spinal cord, is responsible for sensory motor integration and locomotion. There is a long history of studies in insects seeking to understand the neuronal organisation and functions that underlie locomotor behaviour. These studies also describe discrete populations of functionally related spiking and nonspiking interneurons that integrate sensory information to control posture (Malcolm Burrows, 1996), walking (Büschges, Akay, Gabriel, \& Schmidt, 2008) and flight (Robertson, Pearson, \& Reichert, 1982). As in vertebrates, these populations of interneurons share developmental origins with identified populations of interneurons arising from the same neuroblast stem cell (NB). In the locust, for example, midline spiking local interneurons that receive sensory input from the legs to shape the receptive fields of leg motoneurons are all generated from a single NB (Shepherd \& Laurent, 1992) and the unpaired Median NB 
produces a population of GABAergic neurons that respond to sound (Thompson \& Siegler, 1991). Importantly neuronal lineages that have been molecularly characterised in grasshoppers (Jia \& Siegler, 2002; Siegler, Pankhaniya, \& Jia, 2001) and Manduca (Witten \& Truman, 1998) also show that the same principles of developmental organisation are found in insects as are found in vertebrates. In this respect the fact that the orderly generation of molecularly defined neuronal progeny by a lineage-based mechanism provides a unique opportunity to analyse the rules of network assembly.

Drosophila has also proved to be an excellent system in which to extend our understanding of the developmental basis of neuronal circuit assembly because of its life history and the genetic toolset it offers. Its metamorphic life history is advantageous because most of its central neurons are produced in the larval stages during a second neurogenic period (Truman \& Bate, 1988). During this postembryonic phase, NBs divide repeatedly, with the smaller product of each division being a ganglion mother cell (GMC), each GMC divides once to produce two terminally differentiated neurons or glia. The fate of the neurons is determined by Notch signaling, with one sibling activating Notch signaling and the other not; thus, each NB produces two distinct populations of neurons called hemilineages, one that is Notch "On" and the other Notch "Off" (Truman et al., 2010). Using MARCM, Truman et al. (2004) described the organization of the postembryonic neuronal lineages and showed that by the start of metamorphosis the immature progeny of each hemilineage have unique morphology, with neurons from the same hemilineage forming a coherent neuronal population that projects to the same regions of neuropil and suggesting that hemilineages may be functional classes of neurons. Whilst Truman et al. (2004) provided insight into the

larval organization of the postembryonic neurons, they were unable study the hemilineages in adult stages because the neurotactin labeling, used to identify each hemilineage, disappeared early in metamorphosis. In this study we have overcome this limitation by building on the work of Shepherd et al. (2016), who used an antibody to neuroglian to reveal the hemilineage scaffold from larva through metamorphosis and into the adult VNS, to follow every larval lineage through metamorphosis to identify its adult fate.

In the mesothoracic hemineuromere of Drosophila, 25 of the 30 embryonic NBs reactivate to generate a postembryonic lineage. Of the 50 possible hemilineages these NBs could 
produce, 15 are either lost through cell death or do not produce neurons. The mesothoracic hemineuromere therefore contains neurons from 35 different hemilineage pools. There are segmental variations: in the prothoracic hemineuromere there are 23 NBs producing 31 hemilineages and in the metathorax there are 24 NBs producing 32 hemilineages. These 98 thoracic hemilineages account for $90-95 \%$ of the neurons in the adult thoracic VNS. Hemilineages are also units for early molecular diversity in the VNS. Expression of many early transcription factors is restricted along hemilineage lines (Lacin et al., 2014) and many of these transcription factors have homologs involved in fate determination in the vertebrate spinal cord (Thor $\&$ Thomas, 1997). Approaching the analysis of neuronal activity and behavior on the basis of the hemilineage roadmap promises to provide significant steps forward in our understanding of the circuitry that controls movement in insects and other animals. In order to advance our understanding of the functional organization of the hemilineage populations we have generated MARCM clones (Lee \& Luo, 2001) of the thoracic NB lineages in the adult VNS to describe their anatomical organization, to build a detailed understanding of the clonal organization of the VNS neuropil, and to uncover the developmental rules controlling the assembly of the locomotor control circuitry.

\section{Results}

In this paper we used both the postembryonic designation for VNS NB identities (Truman et al., 2004) and the embryonic nomenclature (Schmid, et al., 1999). The nomenclature for postembryonic hemilineages devised by Truman et al. (Truman, et al., 2010) is a notation that describes the NB of origin and whether they are the Notch-on (A) or Notch-off (B) daughter of the GMC division. With the embryonic nomenclature whilst there is agreement in the correspondence between the embryonic and postembryonic designations in most instances, in some cases they are in dispute (Birkholz, et al., 2015; Lacin \& Truman, 2016). In this paper we have used the designations proposed by Lacin et al. (2016). Identification of which hemilineages survive into the adult is based on (Truman et al., 2010). 


\section{Anatomical framework for the adult lineages}

There is a growing literature on the functional organisation of the neuropils of the adult Drosophila VNS that identifies behavioural functions associated with specific neuropil regions. The major neuropil regions and longitudinal tracts used to describe lineage morphology in this paper are summarised in figure 1. By relating lineage morphology to these regions, we can begin to ascribe functional roles for the neurons produced by the lineages. Using this functional anatomy context, we present the lineages in functional groupings, considering lineages with similar functions together. In this project we generated 299 VNS preparations which included at least one identifiable MARCM lineage. From these we had 250 lineages that were suitable for analysis with at least one example of every postembryonic lineage with the exception of lineage 18 (NB 2-4). The number of MARCM clones generated for each lineage is shown in Table 2.

\section{Lineages primarily associated with the leg neuropils}

Merritt and Murphey (1992) defined segmentally repeated ventral regions of VNS neuropil called the leg neuropil, that contains: the sensory afferents from sensory neurons of different sensory modalities that project to different layers within the leg neuropil (Murphey,et al., 1989) with some showing somatotopic representation of the body (Murphey, et al., 1989); the dendrites of leg motorneurons that are organized into a myotopic map (Baek \& Mann, 2009; Brierley et al., 2012). These regions, one for each leg, are the site for the integration of the sensory information required to control leg movements. We have identified 6 of the 22 lineages that have their arborizations restricted to the leg neuropil. We have not included lineages 15 and 24 which generate leg motorneurons and glia and have been described elsewhere (Baek \& Mann, 2009; Brierley et al., 2012; Enriquez et al., 2018; Enriquez et al., 2015).

\section{Lineage 4 (NB 3-1)}

Lineage 4 is present in all three thoracic neuromeres and only the $4 \mathrm{~B}$ hemilineage survives into adult stages. We only generated analysable clones for the pro and metathoracic neuromeres. Lineage 4 neurons are mostly local interneurons with arborizations restricted to the ipsilateral leg neuropil. 
The 4B cell bodies are often spread across the ventral surface of the VNC and do not form a coherent cluster. This tendency is particularly evident in the prothorax (Fig 2a and b). The $4 B$ cells do not produce a coherent primary neurite bundle as they enter the neuropil. The central projections are similar in pro and metathoracic neuromeres, forming a widespread and diffuse projection throughout the ipsilateral leg neuropil (Fig $2 \mathrm{~b}$ and $\mathrm{c}$ ) including dorsal and ventral neuropil. There are major arborizations into primary mechanosensory neuropil (sn, Fig 2e) particularly the anterior and posterior compartments (arrows, Fig 2c). At the anterior of the neuropil there is a distinct and dense arborization in the most ventro-medial neuropil adjacent to VTV and DLV (*, Fig 2c and e) that crosses the midline to produce a small arborization in the same region of the contralateral neuropil. There is also a projection of a small number of larger diameter axonal processes that project into the leg neuropil of 5 of the 6 thoracic hemineuromeres (Fig 2a). At the posterior of the prothoracic neuropil there is a robust projection that runs dorsally ( $d$, Fig $2 a$ and e) to arborize in the ventral and lateral regions of the tectulum ( $d$, Fig $2 e$ ). The projections in the metathorax, like in the prothorax, are largely restricted to the ipsilateral leg neuropil (Fig 2d) with a strong arborization in the primary mechanosensory neuropil (sn, Fig $2 \mathrm{~g}$ ) and a dorsal projection that arborizes in ventral, posterior tectulum ( $d$, Fig $2 b$ and $g$ ). There is also a single intersegmental neuron that projects into the lateral leg neuropil of the other thoracic neuromeres. We did generate MARCM clones for lineage 4 in the mesothorax but all included other clones that confounded analysis, but showed that the mesothoracic lineage is comparable to its pro and metathoracic homologs (data not shown).

\section{Lineage 9 (NB 3-5)}

Lineage 9 is present in all thoracic neuromeres. It was thought that only the $9 \mathrm{~A}$ hemilineage was present in the adult (Truman et al., 2010), but recent studies showed that the contralateral-projecting cells from this lineage are from a truncated 9B hemilineage (Lacin et al., submitted). Both hemilineages innervate the leg neuropil.

The 9 cell bodies form a tight cluster located dorsally; in the prothorax they sit at the anterior of the neuromere adjacent to the neck connectives (arrows, Fig 3a), in the mesothorax they are more lateral (Fig 3a) and in metathorax they are lateral and displaced 
to a more posterior position (arrows, Fig 3a). It is not possible to discriminate the $9 \mathrm{~A}$ and $9 \mathrm{~B}$ cell bodies in the MARCM clones. In all three neuromeres the cells produce a single coherent primary neurite bundle to produce arborizations that are largely restricted to the ipsilateral leg neuromere with a small number of projections crossing the midline or into adjacent neuromeres (Fig 3a). Although the 9A neurons enter the neuropil as a single coherent bundle the projection splits shortly after entering the neuropil to produce two projections, each responsible for different elements of the arborization. This pattern is best seen in the mesothorax but can be seen in the pro and metathoracic projections. In the mesothorax, on entry to the neuropil the primary projection splits to produce two branches (Fig 3a) one turns laterally (9l, Fig 3a) and projects dorsally to produce a diffuse arborization in dorsal leg neuropil. The second projects medially and ventrally (9m, Fig 3a and e) to produce a dense arborization just dorsal to the primary mechanosensory neuropils (sn) (the intermediate neuropil) and invade the $\operatorname{mVAC}(*$, Fig 3a).

The 9B projection enters the neuropil as part of the same bundle as the $9 \mathrm{~A}$ axons and follows the same path as $9 \mathrm{~m}$ projection. As the $9 \mathrm{~m}$ bundle approaches the mVAC the $9 B$ neurites separate from the $9 \mathrm{~m}$ bundle to project medially and ventrally (9B, Fig 3a) to ru under the primary mechanosensory neuropil (sn, Fig 3d), towards the midline to produce an arborization in the ventral-most neuropil, ventral to VT, on either side of the midline (9B, Fig $3 a$ and e).

The details of the 9B projection can be seen in MARCM clones generated using the OK371 driver line which selectively labels the 9B hemilineage (Fig 3g-i). This shows that 9B has extensive bilateral arborizations in ventral neuropil with intersegmental projections, mostly on the contralateral side, that extend into adjacent neuromeres including into the bristle ovoid (arrow, Fig. 3h).

\section{Lineage 10 (NB 2-2)}

Lineage 10 is found in all three thoracic neuromeres and only hemilineage $10 B$ survives in the adult. Although present in all three thoracic segments, we recovered MARCM clones of lineage 10 in only the Pro and mesothorax. Hemilineage 10B produces intersegmental interneurons that arborize in $\mathrm{mVAC}$ and intermediate neuropil. The $10 \mathrm{~B}$ cells form a tight 
cluster close to the ventral midline in the anterior of the neuromere (Fig 3j). The 10B projections are almost identical in both Pro and mesothoracic neuromeres (Fig 3j), the primary neurites enter the neuropil as a single coherent bundle that runs posteriorly to produce a characteristic arborization in ipsilateral leg neuropil constrained to intermediate neuropil and the mVAC (*, Fig 3j-n). The arborization defines the extent of both structures. The primary bundle crosses the midline just posterior to mVAC (arrows, Fig 3j) and produces an arborization in the contralateral neuropil that mirrors the ipsilateral projection but is not as extensive (Fig 3j, $\mathrm{k}$ and I). The projections extend intersegmentally into adjacent neuromeres to arborize in the mVAC and intermediate neuropils (Fig 3I-n). The prothoracic neurons project into both meso and metathoracic neuromeres and the neck connectives (Fig 3j). The mesothoracic neurons project posteriorly into the metathorax and anteriorly into the prothorax and neck connectives (not shown).

\section{Lineage 13 (NB 4-2)}

Both the $13 \mathrm{~A}$ and $13 \mathrm{~B}$ hemilineages survive in the adult and are present in all three thoracic neuromeres (Fig $2 h, k$, and $n$ ). Both the $A$ and $B$ hemilineages produce local interneurons that are restricted to a single hemineuromere.

In the larva, the lineage 13 neurons form a compact cluster but neurons of the two hemilineages are pulled apart as the neuropil expands during metamorphosis with the cells of the $A$ hemilineage lying lateral to those of the $B$ hemilineage (Fig $2 h, k$, and $n$ ). In the prothorax the $13 \mathrm{~A}$ cells sit in a mid-ventral position at the anterior of the neuromere and the $13 \mathrm{~B}$ cells at the anterior midline (Fig $2 \mathrm{~h}$ ). In the mesothorax, the cell positions are similar but the $13 \mathrm{~A}$ cells are more lateral and posterior on the ventral surface (Fig $2 \mathrm{k}$ ). In the metathorax the cells sit more posteriorly in the neuromere with the $13 \mathrm{~A}$ cells in a midventral position and the 13B cells at the midline (Fig $2 n$ ). In some cases, some or all of the 13B cells have been drawn across the midline and sit in the hemineuromere contralateral to their origins (Fig $2 \mathrm{k}$ ).

The central projections of lineage 13 neurons are almost identical in all three neuromeres, the primary projection of $13 \mathrm{~A}$ enters the ipsilateral neuropil directly ventral to the cells 
whereas $13 \mathrm{~B}$ projects contralaterally in a superficial, anterior commissure to enter the contralateral leg neuropil (Fig $2 \mathrm{~h}-\mathrm{p}$ ). Both hemilineages make extensive arborizations in the leg neuropil extending from dorsal to ventral, almost filing the volume (Fig $2 \mathrm{~h}-\mathrm{p}$ ) with the exception of the tectulum and the ovoid both of which are almost devoid of any projections (Fig $2 \mathrm{~m}$ ). It is difficult to completely segregate the arborizations of the two hemilineages but it is evident that both hemilineages are composed of local interneurons that arborize primarily in one hemineuromere with no intersegmental projections. The $13 \mathrm{~A}$ arborizations are restricted to the ipsilateral leg neuropil and do not enter the primary mechanosensory neuropil (sn, Fig 2j, $m$ and p; left leg neuropil). 13A also produces a distinct arborization that extends medially and dorsally to terminate in a medial dorsal region of neuropil (d, Fig $2 \mathrm{i}, \mathrm{j}$, $\mathrm{I}, \mathrm{m}, \mathrm{n}$ and o). The 13B neurons also arborize extensively but in the contralateral leg neuropil but unlike 13A, the 13B neurons arborize extensively in the primary mechanosensory neuropil (sn, Fig 2j, m, p; right leg neuropil). In the metathorax both hemilineages extend their arborizations into the abdominal neuropil (abd, Fig 2p).

\section{Lineage 14 (NB 4-1)}

Lineage 14 is found in all three thoracic neuromeres in the adult. The majority of the neurons in the lineage are hemilineage 14A, the 14B hemilineage includes only a single neuron (arrowheads, Fig 3t). Hemilineage 14A produces local interneurons that are restricted to the contralateral leg neuropil.

The 14A cells sit adjacent to the ventral midline in the anterior of the neuromere although in the metathorax the neurons are more posterior (T3, Fig 3t). In some circumstances some or all 14A cells can be drawn across the midline to sit contralateral to their origin, as seen for the mesothorax in Fig 3t. The 14A projections are the same in all thoracic neuromeres (Fig 3t-w), the primary neurites project contralaterally in an anterior and superficial tract to arborize extensively in the contralateral leg neuropil (Fig 3t-w). All of the neurons make the same basic projections within the neuromere with no projections into adjacent neuromeres. The neurons produce extensive fine dendritic arborizations in the primary mechanosensory neuropil (sn, Fig $3 u-w)$ with more robust and varicose projections in more dorsal regions of leg neuropil. 
The single 14B cell body is separated from 14A cells and sits more laterally (arrowheads, Fig 3t). The primary neurite projects dorsally to produce a large arborization of fine branches in ipsilateral leg neuromere before crossing the midline dorsally and to produce a varicose axonal projection in contralateral leg neuropil, that mirrors the ipsilateral arborization (14A, Fig $3 u$ and $v)$.

\section{Lineage 23 (NB 7-4)}

Lineage 23 is found in all three thoracic neuromeres, although we recovered MARCM clones in just the pro and mesothoracic neuromeres. Only the 23B hemilineage survives in the adult. The 23B central projections are the same in pro and mesothoracic neuromeres producing interneurons that arborize in ipsilateral leg neuromere and project into contralateral neuropil.

The 23B cells are amongst the most dorsally located somata in the VNS and form a discrete cluster on the dorsal surface in the posterior lateral quarter of the neuromere (arrowheads, Fig $3 p-s)$. The primary neurites make a long and sweeping projection extending dorsally and medially around the outer edge of the tectulum. As the neurites near the midline they arborize profusely to create a large arborization that spans the anterior to posterior extent of the ipsilateral leg neuropil (Fig 3p-s). The arborizations are restricted to the ventral neuropil occupying most of the primary mechanosensory neuropil (sn, Fig 3q-s).

The neurons extend axons across the midline in a posterior commissure to form a small region of varicose projections (Fig 3p). The contralateral projections from prothoracic neurons extend anteriorly and posteriorly to terminate in posterior prothoracic neuropil and the ovoid (T1, Fig $3 p$ and $r$ ). The contralateral projection from the mesothoracic neurons project anteriorly in the DLV tract to the ovoid (T2, Fig 3p and $r$ ) and posteriorly in DLV to terminate just inside the metathoracic neuromere (Fig $3 p$ and $s$ ). Both the prothoracic and mesothoracic hemilineages produce an ipsilateral projection that arborizes in the ovoid (T1i and $\mathrm{T} 2 \mathrm{i}$, Fig $3 \mathrm{p}$ and $\mathrm{r}$ ). 


\section{Lineages primarily associated with wing neuropil}

As with the leg there are regions of VNS neuropil that are associated with control of wing movements and flight. Most of the wing circuitry is in the tectulum (Power, 1948) which occupies the dorsal regions of the VNS and has no obvious segmental sub-divisions. It sits over the mesothoracic neuromere and posterior parts of the prothoracic neuromere and anterior of the metathoracic neuromere. The tectulum is further sub-divided into functional domains with the dorsal-most regions responsible for flight motor control and the more ventral regions responsible for leg and wing coordination (Namiki et al., 2018). There is also an anterior posterior segregation of function with anterior regions responsible for neck motor systems, the middle sections for wing control and the posterior for haltere control. Additionally, the ovoid, that sits at the interface of the pro and mesothoracic neuromeres and is a dense neuropil that receives afferent inputs from mechanosensory neurons on the notum and wings. Despite the obvious importance of flight and wing motor control only 3 lineages uniquely target the tectulum and the ovoid.

\section{Lineage 2 (NB 2-1)}

Lineage 2 is found in all three thoracic neuromeres (Fig 4a-c) and in abdominal neuromeres (data not shown), and only the $2 \mathrm{~A}$ hemilineage survives to adulthood. The projections of the $2 \mathrm{~A}$ neurons are similar in all neuromeres (Fig $4 \mathrm{a}-\mathrm{i}$ ) producing interneurons that arborize dorsally and project into adjacent neuromeres.

The $2 \mathrm{~A}$ cells sit on the ventral midline at the anterior of the neuromere (Fig 4a-c). The primary projection forms a single bundle that extends to dorsal neuropil where it arborizes extensively in ipsilateral tectulum (Fig 4a-i) around MTD and HT. The arborizations are mostly restricted to medial ipsilateral tectulum extending through the full anterior posterior extent of the neuromere. There are minor projections into contralateral neuropil arborizing in the same regions as ipsilateral. (Fig 4a-c). The arborizations in the pro and mesothoracic neuromeres project into and arborize extensively in dorsal anterior tectulum (Fig 4e and f). The metathoracic neurons extend anteriorly to overlap with its mesothoracic homolog in the tectulum (Fig $4 \mathrm{~g}$ and $\mathrm{h}$ ). 


\section{Lineage 6 (NB 5-2)}

Lineage 6 is present in all three thoracic neuromeres with both $6 \mathrm{~A}$ and $6 \mathrm{~B}$ hemilineages surviving in the adult. The lineages they produce are some of the more complex and diverse lineages in the VNS with considerable segmental variation and are best described separately (Fig 5).

In the prothorax the $6 \mathrm{~A}$ and $\mathrm{B}$ somata form a large cluster on the ventral surface running the width of the neuromere at the posterior of the neuromere (arrowheads, Fig 5a and c). The two hemilineages form separate primary neurite bundles with the $6 \mathrm{~A}$ bundle lateral to that of $6 \mathrm{~B}$ (Fig $5 \mathrm{c}$ ). The $6 \mathrm{~A}$ bundle enters the neuropil and projects dorsally without branching into dorsal tectulum to produce an extensive ipsilateral arborization that wraps around MTD and HT (Fig. 5c) with arborizations extending into prothoracic neuropil (6A, Fig $5 b)$, dorsal anterior tectulum around MTD and HT (6A, Fig $5 \mathrm{~d}$ ) and anterior mesothoracic neuropil (Fig 5a). The 6A primary projection also crosses the midline (small arrow, Fig $5 \mathrm{c}$ ) to form a less extensive arborizations in the contralateral dorsal neuropil around MTD and HT mirroring the ipsilateral arborization (Fig 5c). This contralateral projection extends anteriorly and posteriorly in the HT. The anterior projection arborizes in dorsal prothoracic neuropil (6Ac, Fig 5b) and enters the contralateral cervical connective. The posterior projection extends through the mesothorax and into the metathorax (6Ac, Fig 5e) producing arborizations in dorsal neuropil as it descends. In the mesothorax it produces a distinct lateral varicose branch ( $\mathrm{Ib}$, Fig $5 \mathrm{a}$ and $\mathrm{d}$ ) that projects to dorso-lateral tectulum. In the metathorax, the projection terminates in a laterally curving arborization of large varicose processes in both posterior tectulum and anterior leg neuropil (tp, Fig 5a and f). 6A also produces a projection that extends laterally and ventrally to form a varicose projection into the lateral leg neuropils (LL, Fig $5 a, b$ and f).

The $6 \mathrm{~B}$ neurons extend medially across the midline in a mid-dorsal/ventral plane (6B, Fig5c) to form a complex and almost symmetrical arborization either side of the midline, restricted to anterior tectulum between DLV and HT and ventral to the 6A arborizations (Fig 5c). 6B also projects bilaterally into the ovoid $(6 \mathrm{~B}, \mathrm{Fig} 5 \mathrm{~d})$. There is some overlap between the $6 \mathrm{~A}$ and B arborizations and it isn't possible to completely discriminate the two hemilineages. 
In the mesothorax the $A$ and $B$ cells are separated with the $6 \mathrm{~A}$ somata on the posterior/medial edge of the neuromere, medial to the leg nerve $(6 \mathrm{~A}, \mathrm{Fig} 5 \mathrm{~g})$ and the $6 \mathrm{~B}$ somata more anterior on the midline in a mid-neuromere position (6B, Fig 5g). The 6A primary neurites run laterally and dorsally without branching to dorsal tectulum (6A, Fig $5 \mathrm{j}$ ) to form an extensive arborization in ipsilateral dorsal tectulum around MDT and HT (6A, Fig $5 \mathrm{j})$, before crossing the midline to arborize in the same area of the contralateral tectulum (Fig 5j). The 6A projections extend anteriorly and posteriorly in $\mathrm{HT}$ to arborize both ipsi and contralaterally in anterior and posterior tectulum (Fig 5h-k).

The $6 \mathrm{~B}$ neurons project medially to cross the midline in the mid-ventral plane, producing an extensive arborization either side of the midline in ventral tectulum, ventral to DLV and HT and $6 \mathrm{~A}$ arborizations (6B, Fig 5j). The 6B projection extends anteriorly (arrowheads, Fig 5g) where it arborizes in lateral tectulum and produces a characteristic ventral arborization between the ovoids either side of the midline $(6 \mathrm{bm}$, Fig $5 \mathrm{i})$. There is some overlap between the $6 \mathrm{~A}$ and $\mathrm{B}$ projections.

The metathoracic lineage 6 has a simpler structure. The A and B cells sit together at the midline in the posterior neuromere with $6 \mathrm{~B}$ somata medial to those of $6 \mathrm{~A}$ (Fig 5I). The 6B cells are fewer in number than in pro and mesothoracic neuromeres. The $6 \mathrm{~A}$ neuron projections extend anteriorly with a long arcing projection that enters the neuropil at the anterior of the metathoracic neuromere and crosses the midline (Fig $5 \mathrm{I}$ ). The 6A arborizations are displaced anteriorly to posterior tectulum extending anteriorly to mid tectulum (Fig $5 \mathrm{I}$ and $\mathrm{m}$ ). The ipsilateral projection is compact and dense whereas the contralateral arborization is less extensive and more diffuse (Fig $5 \mathrm{I}$ ). As in the pro and mesothorax the projections are restricted to dorsal tectulum. The $6 \mathrm{~A}$ projection also extends anteriorly in HT to the anterior tectulum ipsilaterally (Fig $5 \mathrm{~m}$ ) and contralaterally (not shown). The 6A neurons also produce a small varicose projection into lateral leg neuropil (LL, Fig 5m).

The $6 \mathrm{~B}$ primary neurites are few and project anteriorly, medial to the $6 \mathrm{~A}$ bundle, to cross the midline at the anterior of the metathoracic neuropil and form a small entirely contralateral arborization constrained to ventral tectulum (6B, Fig 5n). 


\section{Lineage 11 (NB 7-2)}

Lineage 11 is found in only in the pro and mesothoracic neuromeres. In the prothoracic neuromere only the $11 \mathrm{~A}$ hemilineage survives to adult but in the mesothorax both $11 \mathrm{~A}$ and B hemilineages survive (Truman et al. 2009). We only generated analysable MARCM clones for the mesothoracic lineage 11 which produces local interneurons restricted to dorsal neuropil of the hemineuromere of origin.

The $11 \mathrm{~A}$ and $\mathrm{B}$ cells are on the dorsal surface at the posterior of the neuromere (Fig $4 \mathrm{j}$ ) and do not form a coherent cluster but are "smeared" across the dorsal surface (arrows, Fig4j). It is not possible discriminate the A and B cells but it is possible to discriminate their primary neurite bundles. The $11 \mathrm{~A}$ primary neurites enters the neuropil from dorsal and lateral to the 11B bundle, projecting ventrally before curving to turn dorsally and project into dorsal tectulum (11B, Fig $4 \mathrm{j}-\mathrm{I})$ to produce a dense arborization crossing the midline into contralateral tectulum (11A, Fig 4I). The 11B neurites form a more robust fascicle (11B, Fig $4 j-1)$ that enters the neuropil dorsally and projects ventrally and medially curving around the tectulum producing an arborization of fine process in the dorsal leg neuropil and ventral tectulum. There is a further $11 \mathrm{~B}$ arborization in ventral leg neuropil including a small arborization in the mechanosensory neuropil (sn, Fig 4I). The main neurite bundle also projects anteriorly in DLV and to form a dense, but restricted, arborization close to the midline between DLV and MTD and into mechanosensory neuropil of the ovoid (11B, Fig $4 k)$.

\section{Lineages associated with leg and wing neuropils}

The majority (13 of 22) lineages described are not restricted to only leg or wing neuropil but contribute to both.

\section{Lineage 0 (MNB)}

Lineage 0 is present in all three thoracic neuromeres but only the $A$ hemilineage survives to adult stages. The $\mathrm{OA}$ neurons have the same basic structure in each thoracic neuromere with slight segmental differences (Fig 6a-i) producing unpaired, bilaterally symmetrical local interneurons. 
The $O A$ cells sit on the ventral midline in a tight cluster in all three neuromeres (Fig 6a). The cells produce a single robust primary neurite bundle that projects dorsally along the midline producing a complex, bilaterally symmetrical arborization that is largely restricted to the neuromeres of origin but with some extensions into adjacent neuromeres (Fig 6a). In all three neuromeres the bulk of the dense bushy arborizations are in leg neuropil and specifically in the mVAC and intermediate neuropil resembling the 10A arborizations (Fig 6ai). There is a small arborization into the leg primary mechanosensory neuropils (sn, Fig 6b, $f$ and i) and for the pro and mesothoracic hemilineages the ovoid (Fig $6 \mathrm{c}$ and e). In the pro and mesothorax a projection extends dorsally to arborize in the tectulum (Fig $6 \mathrm{c}$ and f), the arborization is bilayered with distinct ventral arborizations, ventral to MTD, ITD and HT (OV, Fig 6f) and dorsal arborizations, dorsal to MTD, ITD and HT (Od, Fig 6f). The prothoracic neurons project posteriorly in DLV to terminate in ventral parts of the anterior tectulum and the ovoid (arrow, Fig 6a and c). The mesothoracic neurons projects anteriorly and posteriorly in DLV into the ventral parts of the anterior tectulum, the ovoid, the prothoracic mVAC and the metathoracic neuropil (Fig 6a-i). The metathoracic neurons extend anteriorly in DLV to terminate with a varicose arborization in mesothoracic mVAC. Where projections enter adjacent neuromeres they overlap the arborizations of their segmental homolog.

\section{Lineage 1 (NB 2-1)}

Lineage 1 is found in all three thoracic neuromeres and both $A$ and $B$ hemilineages survive to adult stages (Fig 6j-r). Unlike other thoracic lineages, the A and B cells are found in different neuromeres, the $1 \mathrm{~A}$ cells remain in the neuromere of origin (Fig $6 \mathrm{j}$ ) but the $1 \mathrm{~B}$ cells are pulled into the next anterior neuromere during metamorphosis (Fig 6p). Hemilineage $1 \mathrm{~A}$ produces a distinctive population of local interneurons that project ipsilaterally and contralaterally in leg and wing neuropils. Hemilineage 1B produces a population of local interneurons restricted entirely to ipsilateral, ventral leg neuropil.

The $1 \mathrm{~A}$ cell bodies are located dorsolateral at the anterior of the neuromere. In all three neuromeres the primary neurites project ventromedially to enter the neuropil in the anterior lateral corner. Ipsilateral to their somata, the $1 \mathrm{~A}$ neurons produce an extensive 
arborization of fine processes. The primary projection crosses the midline in a superficial, ventral commissure to produce a significant varicose projection in contralateral leg neuropil.

In the prothorax the $1 \mathrm{~A}$ projection is distorted and the contralateral axonal process turns abruptly anterior on entry to the neuropil looping ventrally under the MTD tract and the cervical connective (Fig 6j). The ipsilateral dendrites form a two-pronged projection. The more lateral projection (1l, Fig $6 \mathrm{j}$ ) extends posteriorly and laterally to arborize in a midneuropil region at the interface of the leg neuropil and the anterior tectulum, dorsal to DLV (1l, Fig $6 \mathrm{j}$ and $\mathrm{k}$ ). The medial projection ( $1 \mathrm{~m}$, Fig $6 \mathrm{j}$ and $\mathrm{k}$ ) extends posteriorly and arborizes in the posterior-medial quarter of prothoracic neuropil and ventro/medial regions of the anterior tectulum but dorsal to the ovoid (1m, Fig $6 \mathrm{j}$ and $\mathrm{k}$ ). Fine $1 \mathrm{~m}$ processes also cross the midline (*, Fig $6 \mathrm{j})$ to form a compact bilaterally symmetrical projection around to the midline ( $1 \mathrm{~m}$, Fig $6 \mathrm{j}$ and $\mathrm{k}$ ). Contralaterally the primary projections arborize extensively creating a compact arborization in the mid prothoracic leg neuropil (1c, Fig $6 \mathrm{j}$ and $\mathrm{k}$ ).

In the mesothorax the $1 \mathrm{~A}$ projections are more extensive than in the pro and mesothorax (Fig 6j). Ipsilateral the primary process produces three distinct arborizations (1a, $1 \mathrm{~m}$ and $1 \mathrm{p}$, Fig $6 \mathrm{j})$. The $1 \mathrm{a}$ arborization extends anteriorly (1a, Fig $6 \mathrm{j}$ and $\mathrm{I}$ ) into ventral parts of the anterior tectulum just lateral to MTD and just dorsal to the ovoid (1a, Fig 6k). The medial arborization $(1 \mathrm{~m})$ projects dorsally and medial to the tectulum where it ramifies across the full width of the mid regions of the tectulum, just dorsal to MTD and ITD, and into medial leg neuropil at the interface with the tectulum ( $1 \mathrm{~m}$, Fig $6 \mathrm{~m})$. The $1 \mathrm{~m}$ arborization also extends into anterior tectulum between DLV and MTD but medial to the $1 \mathrm{a}$ arborization $(1 \mathrm{~m}$, Fig $6 \mathrm{j}$, I and $\mathrm{m}$ ). The $1 \mathrm{p}$ branch (1p, Fig $6 \mathrm{j}$ ) extends posteriorly, curving medially into ipsilateral leg neuropil where it branches extensively in the medial regions between DLV and VLT (1p, Fig 6j and $\mathrm{m})$.

Contralaterally the primary projection extends to form two arborizations. The more medial arborization $(1 \mathrm{~cm})$ runs dorsally and ventrally $(1 \mathrm{~cm}$, Fig $6 \mathrm{j}$ and $\mathrm{m})$ to form an array of fine processes in leg neuropil that mirrors the ipsilateral $1 p$ arborizations. The primary projection extends to the lateral leg neuropil before turning obliquely to form a varicose arborization $(1 \mathrm{cl}$, Fig $6 \mathrm{~m})$ in lateral regions of the anterior leg neuropil. 
In the metathorax the $1 \mathrm{~A}$ projection enters the neuropil and splits to form three arborizations that correspond to those seen in the mesothoracic neurons. The first (1a, Fig $6 j$ ) extends anteriorly and dorsally into lateral tectulum (1a, Fig 6j and $n$ ), the second (1m, Fig 6j) projects across the midline and ramifies through ventral regions of posterior tectulum $(1 \mathrm{~m}$, Fig $6 \mathrm{~m})$ extending into more anterior tectulum to overlap its mesothoracic homolog. The third arborization (1p, Fig 6j) extends posteriorly into the anterior lateral leg neuropil, dorsal to DLV (1p, Fig 6o). The primary axonal process crosses the midline to form varicose terminals in the anterior lateral quarter of leg neuropil (1c, Fig 6j and o).

The $1 \mathrm{~B}$ neurons in meso and metathorax survive to the adult stage but in $\mathrm{T} 1$ they do not. The mesothoracic 1B neurons sit in the posterior lateral quarter of the prothoracic neuromere (Fig 6p) and the metathoracic neurons sit in posterior medial quarter of the mesothorax (Fig 6p). The 1B hemilineage in the first abdominal neuromere also survives and projects into metathoracic neuromere (data not shown). The pro and mesothorax $1 \mathrm{~B}$ projections are the almost identical, creating a diffuse projection throughout ipsilateral leg neuropil restricted almost exclusively to ventral neuropil in the primary mechanosensory neuropil and mVAC (sn, mVAC Fig $6 q$ and $r$ ) with no projections across the midline or into adjacent neuromeres. A few lateral projections extend dorsally (arrowheads, Fig 6q and r) into lateral leg neuropil.

\section{Lineage 3 (NB 7-1)}

Lineage 3 is present in all three thoracic neuromeres and both $A$ and $B$ hemilineages survive in adult. The basic organization of the $3 \mathrm{~A}$ and $\mathrm{B}$ projections are largely the same in all three thoracic neuromeres with the $3 \mathrm{~A}$ neurons projecting into leg neuropil and the $3 \mathrm{~B}$ neurons projecting into the tectulum (Fig 7a-h).

In the prothorax the lineage 3 cells form a tight cluster located ventro-laterally at the posterior of the neuromere (Fig 7a) but it isn't possible to discriminate the A and B somata. The $3 A$ and $B$ neurons enter the neuropil as a single primary neurite bundle but on entry to the neuropil the bundle bifurcates (Fig 7a). The 3A projection turns ventrally to produce an extensive arborization of fine branches that ramify throughout ipsilateral leg neuropil from anterior to posterior and dorsal to ventral (3A, Fig 7a and b), including the primary 
mechanosensory neuropil, but not the mVAC (sn, mVAC, Fig 7b). The $3 \mathrm{~A}$ arborizations remain ipsilateral and do not cross the midline.

The 3B primary projection turns medially and dorsally and projects to the dorsal most regions of the tectulum (Fig 7a and c). Viewed dorsally the 3B projection creates a characteristic 4 pronged "yoke" shaped arborization with: a medially directed arborization (3bm, Fig 7a) that crosses the midline to dorsal tectulum, dorsal to MTD and HT (Fig 7c); an anterior arborization (3ba, Fig 7c) in the anterior tectulum (a, Fig 7b); a posterior arborization ( $3 \mathrm{bp}$, Fig 7c) into ventral tectulum, ventral to MTD and HT (3bp, Fig 7a) and a lateral arborization (3bl, Fig 7a) that projects laterally and ventrally to a region of neuropil just dorsal to the ovoid (3bl, Fig 7c).

In mesothorax the $3 \mathrm{~A}$ and $\mathrm{B}$ cells are located ventro-medially at the posterior of the neuropil "smeared" across the ventral surface such that some cells sit in the metathoracic neuromere (arrows, Fig 7d). Their primary neurites fasciculate into a robust bundle that enters the neuropil from a posterior medial position (black arrow, Fig 7d) and projects dorsally and laterally before bifurcating (*, Fig 7e). The A bundle turns laterally and ventrally to produce a large and diffuse arborization that almost completely fills the ipsilateral leg neuropil including the primary mechanosensory neuropil (sn, Fig 7e). The B bundle turn medially and dorsally to the tectulum to produce a dense arborization in the dorsal most ipsilateral tectulum, dorsal to MTD and ITD, (Fig 7e) with a minor projection crossing the midline into contralateral tectulum.

In the metathorax the A and B cells sit ventromedially at the posterior of the neuromere and are widely spread (Fig 7f). Their primary neurites combine in a that enters the neuropil and then bifurcates. The A neurons form a characteristic posteriorly turning loop (inset, Fig 7f) that projects into ipsilateral leg neuropil where they form an arborization that fills the leg neuropil including the primary mechanosensory neuropil (sn, Fig 7h). The 3B neurons project anteriorly (3B, Fig $7 f$ and $g$ ) to produce a small and compact arborization in lateral regions of posterior tectulum (Fig 7g). The metathoracic 3B hemilineage has significantly fewer cells that its pro and mesothoracic homologs.

Lineage 5 (NB 5-3) 
Lineage 5 is found in all three thoracic neuromeres but only the $B$ hemilineage survives to adult stages. We only generated one analysable clone for the prothorax, but other evidence indicates that meso and metathoracic lineage 5Bs are comparable (data not shown). The 5B neurons produce a complex population of intersegmental interneurons that project to all thoracic and some abdominal neuromeres.

The 5B cells sit at the ventral midline at the posterior of the neuromere (arrows, Fig 7i). The cells produce a robust primary neurite bundle that enters the neuropil close to the midline (arrow, Fig 7k) and immediately crosses the midline to produce extensive arborizations in the contralateral hemineuromere (Fig 7i-I). Most of the arborizations are in primary mechanosensory neuropil (sn, Fig 7j) but extend dorsally at the midline into anterior tectulum (Fig 7j). Ipsilateral to the cells there is a less extensive arborization in medial parts of primary mechanosensory neuropil (arrow, Fig 7j) and a dorsal arborization in anterior tectulum that mirrors the contralateral arborization.

The $5 \mathrm{~B}$ neurons also have extensive intersegmental projections extending into the meso and metathorax and the abdominal neuromeres (Fig 7i). At the interface of the T1 and T2 neuromeres the arborization is almost symmetrical around the midline (Fig 7k), with most of the arborization in the ovoid (Fig $7 \mathrm{k}$ ) but also extending laterally in mid-level regions of the tectulum. In the mesothorax majority of the arborizations are in the contralateral mechanosensory neuropil (sn, Fig 7l) with a lesser ipsilateral projection in ipsilateral mechanosensory neuropil. There is also a dorsal arborization into medial regions of ventral tectulum, dorsal to DLV (Fig 7l). In the metathorax the arborizations are again restricted to mechanosensory neuropil with the contralateral projection more significant than ipsilateral (sn, Fig $7 \mathrm{~m})$. There are also arborizations in the mechanosensory neuropils of the abdominal neuromeres (abd, Fig 7m). There are also axons ascending into the SEG via the cervical connectives (Fig 7i).

\section{Lineage 7 (NB 3-2)}

Lineage 7 is found in all three thoracic neuromeres but only the $B$ hemilineage survives to the adult stage. We generated clones for the meso and metathorax, but it is present in the prothorax as described by Brown and Truman (2009). 
In the mesothorax the 7B cells form a tight cluster in the anterior of the neuromere in a mid-ventral position (arrowheads, Fig 8a). The primary neurites form a strong fascicle that projects dorsally and medially crossing the midline in the commissure of the mesothoracic neuromere $(\mathrm{CMN})$ just posterior to the hemilineage $2 \mathrm{~A}$ tract (Fig $8 \mathrm{a}$ and $\mathrm{c}$ ) arborizing both sides of the midline.

Ipsilateral to the somata the primary neurite produces two arborizations of fine fibrous branches. The more lateral arborization extends dorsally to arborize through the anterior and mid tectulum (bi, Fig 8a-d). The second arborization is medial to bi and extends posteriorly and ventrally to straddle the midline in the medial regions of leg neuropil between the DLVs (biv, Fig 8 a, $c$ and d). As the primary axon bundle crosses the midline it generates a discrete set of branches that extend to the dorsal most regions of the tectulum to spread across the dorsal surface both sides of the midline (bd, Fig 8c). There is also a characteristic varicose projection that extends laterally to terminate in the dorsolateral leg neuropil (bl, Fig 8a and d).

The contralateral arborizations are less extensive but mirror the ipsilateral arborizations. After crossing the midline, the primary projection extends laterally before bifurcating to form an ascending and descending projection (bca and bcp, Fig 8a). The ascending projection extends into ipsilateral anterior tectulum and through the ipsilateral cervical connective. The posterior projection extends into posterior tectulum. As these ascending and descending projections pass though the neuromeres they produce a robust, varicose projection in all three thoracic neuromeres that extends laterally to terminate in the dorsolateral leg neuropil (*, Fig $8 \mathrm{a}, \mathrm{b}, \mathrm{d}$ and e), mirroring the $7 \mathrm{bl}$ projection in ipsilateral mesothorax. There is also a small but discrete projection that extends dorsally and laterally to create a small, distinct arborization in dorsal tectulum (bcd, Fig 8a and c).

In the metathorax the 7B cells are not as tightly clustered as in the mesothorax and spread across the antero-medial margin of the ventral surface of the neuromere (arrowheads, Fig 8f). The primary neurite projection forms a single cohesive bundle the projects anteriorly and then medially to cross the midline as part of the Haltere Commissure producing dense 
arborizations both sides of the midline (Fig 8f). Ipsilateral to the cells the arborization forms a dense and bushy arborization restricted to posterior tectulum with a few fibres extending into more anterior tectulum (bi, Fig 8f). Contralateral to the 7B cells the arborizations are more robust in structure but not as extensive with a small projection restricted to the posterior tectulum (bc, Fig $8 \mathrm{f}$ and i). There are also ascending fibres in the HT that extend varicose processes in the lateral tectulum as they ascend to terminate in anterior tectulum (bca, Fig 8f-i).

\section{Lineage 8 (NB 3-3)}

Lineage 8 is found in all three thoracic neuromeres with both the $A$ and $B$ hemilineages surviving to the adult stage. The $A$ hemilineage produces neurons with local arborizations in the ipsilateral leg neuropil and the $B$ hemilineage produces intersegmental neurons that arborize in the tectulum.

Prothorax - In the prothorax the A and B cells form distinct clusters on the ventro-medial surface at the anterior of the neuromere close to the neck connective (Fig 9a). The primary neurites of the two hemilineages form distinct bundles that enter the neuropil at different points with $A$ entering lateral to $B(8 \mathrm{~A}$ and $8 \mathrm{~B}$, Fig 9a). The $A$ primary neurites project dorsally and curve laterally to produce a dense bushy arborization in dorsal and lateral ipsilateral leg neuropil (8A, Fig 9a and b). The arborization extends throughout the leg neuropil but with the densest arborizations in the dorsal regions (Fig 9b). There is also a medially directed arborization that terminates ipsilaterally in the dorsal anterior tectulum (8A, Fig 9b).

The $8 \mathrm{~B}$ primary neurites enter the neuropil adjacent to the neck connective and project posteriorly and medially to cross the midline with a distinct U-shaped projection that turns anteriorly to enter the contralateral cervical connective (8B, Fig 9a). As the axons curve and cross the midline they produce a fan shaped arborization that extends posteriorly (Fig 9a) along both sides of the midline in anterior tectulum (Fig 9b) with the ipsilateral processes being more robust. The $8 \mathrm{~B}$ arborization also extends ventrally into leg neuropil (arrowhead, Fig 9b) and into the ovoid and the tectulum just dorsal to the ovoid (arrowhead, Fig 9c). 
Mesothorax - In the mesothorax the A and B cells form separate clusters on the ventral surface with the A cells at the anterior lateral edge of the neuromere ( $8 \mathrm{~A}$, Fig $9 \mathrm{~d})$ and the $B$ cells more medial in a mid-ventral position ( $8 \mathrm{~B}$, Fig $9 \mathrm{~d})$. The $A$ and $B$ primary neurites enter the neuropil separately. The A neurites enter the neuropil laterally and project dorsally and medially to produce to distinct arborizations. The first arborizations turns sharply laterally to produce a dense arborization in dorsal and lateral regions of the leg neuropil (al, Fig 9d and g). The second extends dorsally and medially to arborize in the dorsal most part of the ipsilateral tectulum (ad, Fig 9g).

The 8B primary neurites enter the neuropil from ventral and form a robust dorsomedial directed bundle (Fig 9d). After entering the neuropil, the neurons turn abruptly medial and split to produce four projections. The first projection (bi, Fig 9d) remains ipsilateral and projects both anteriorly and posteriorly. The posterior bi projection forms a dense arborization in ipsilateral ventral leg neuropil, including the intermediate and primary mechanosensory neuropil (bip, Fig 9g). The anterior bi projection arborizes in the ipsilateral prothoracic intermediate neuropil (bia, Fig 9e). The second projection (bm) is medial to bi and projects anteriorly and ventrally into the tectulum (bm, Fig 9f). The third projection (bcl) crosses the midline in the commissure of the mesothoracic neuromere without branching and projects anteriorly and posteriorly in the $\mathrm{HT}$ (bcl, Fig 9d-g). The anterior portion terminates with large varicose, arborizations in lateral anterior tectulum (bcl, Fig 9e), while the posterior portion produces a robust varicose projection in the ventral tectulum over the mesothorax (bcl, Fig 9g) and in contralateral metathoracic leg neuropil (bcl, Fig 9h). The fourth projection ( $\mathrm{bcm}$ ) crosses the midline and immediately turns posteriorly in MTD to produce a varicose arborization in the dorsal contralateral tectulum (bcm, Fig $9 f$ and g). In metathorax the A and B somata are closely associated and it isn't possible to resolve the two populations but the $8 \mathrm{~A}$ cells are more posterior (Fig 9i). The somata lie on the lateral edge of the VNS in a mid-neuromere position (Fig 9i). The two clusters produce a single primary neurite bundle that runs anteriorly under the cell body cortex but superficial to the neuropil (arrowheads, Fig 9i). The 8A primary neurites enter the neuropil towards the anterior of the metathoracic neuromere as it turns dorsal (Fig 9m) to produce a dense 
arborization in the lateral dorsal regions of the ipsilateral leg neuropil (8A, Fig $9 \mathrm{~m})$ before extending medially and arborizing in the medial and ventral leg neuropil (8A, Fig 9m).

The $8 \mathrm{~B}$ primary neurites continue an almost linear anterior projection to enter the neuropil at the intersection point of the Meta and mesothoracic neuromeres (arrow, Fig 9i and I). On entry the neurite bundle splits into four. The first projection (bi) separates upon entry to the neuropil to produce a short ipsilateral, dorsally directed process that produces an arborization that almost fills the posterior ipsilateral tectulum, spreading into the medial regions of the tectulum over the mesothorax (bi, Fig 9l). The remaining projections all cross the midline in the haltere commissure. The second projection (bcv) is a minor projection and is the most posterior and ventral of the $8 \mathrm{~B}$ projections as it crosses the midline and produces small varicose arborization in ventral tectulum (bcv, Fig 9i and I). The third projection (bcap) crosses the midline and bifurcates midway across the neuromere to extend anteriorly and posteriorly in HT producing a contralateral varicose arborization that mirrors the 8bi arborizations (bcap, Fig 9i and I). The fourth projection (bc) is the most anterior of $8 \mathrm{~B}$ commissural processes and after crossing the midline turns anteriorly in the MTD before splitting to produce a parallel projection in the MDT, with both producing arborizations in the anterior regions of dorsal tectulum (bc, Fig 9i, $\mathrm{k}$ and I). The 8bc group includes the ChINs (Strausfeld \& Seyan, 1985) that receive direct input from haltere afferents.

\section{Lineage 12 (NB 6-1)}

Lineage 12 is found in all three thoracic neuromeres and both the $A$ and $B$ hemilineages survive into adult in pro and mesothorax but in the metathorax the 12A hemilineage dies. The anatomy of the $12 \mathrm{~B}$ neurons is consistent in all three neuromeres but the $12 \mathrm{~A}$ neurons shows significant segmental differences.

Prothorax - In the prothorax the lineage 12 cells sit on the ventral surface of the VNS at the posterior of the neuromere, and the A and B somata are readily distinguished with $12 \mathrm{~A}$ lateral to $12 \mathrm{~B}$ (Fig 10a). The primary neurites from the two hemilineages form distinct bundles. The $12 \mathrm{~A}$ bundle enters the neuropil from a ventral lateral position and projects dorsally and laterally before turning medially (12A, Fig 10a and c). Shortly after the medial turn the primary bundle splits into three projections. The first projection turns laterally from 
the main branch and curves around the outer edge of the tectulum to produce a robust varicose arborization in the dorsal/lateral anterior tectulum (aid, Fig 10c). The second projection (aia) heads medially to DLV and runs anteriorly into the prothorax producing a complex arborization in intermediate neuropil (aia, Fig 10a-c). At the anterior of the prothoracic neuropil the aia projection crosses the midline, enters the contralateral DLV and produces an arborization that mirrors its ipsilateral arborization. This creates a characteristic “A-Frame' shaped projection viewed from dorsal (Fig 10a). The third projection (ad) is a continuation of the primary projection and extends dorsally and medially (ad, Fig 10c) into the tectulum, where it splits to produce a robust varicose arborization in the dorsomedial tectulum that crosses the midline (ad, Fig 10d), and a second arborization in the ventral tectulum that remains ipsilateral (12adv, Fig 10d).

The $12 \mathrm{~B}$ neurons enter the neuropil medial to $12 \mathrm{~A}$ and run medially to form a robust arborization that crosses the midline ventral to the tectulum in a posterior commissure (Fig 10a and c). The main projection extends anteriorly and diagonally across the contralateral leg neuropil (Fig 10a) to terminate in a dense arborization of fine processes in the intermediate neuropil and the primary mechanosensory neuropil (sn, Fig 10b and c) of the contralateral leg neuropil. The 12B arborization overlaps with the some of the ventral branches of the $12 \mathrm{~A}$ projections but the $12 \mathrm{~B}$ arborizations tend to be more ventral and lateral (Fig 10b). The 12B projection also produces two minor branches, either side of the midline that extend into the mechanosensory neuropils to produce a diffuse but varicose projection (arrows, Fig 10b).

Mesothorax - In the mesothorax the lineage 12 cells sit at the posterior medial edge of the neuromere but are spread across the ventromedial quarter of the neuromere (Fig 10e). The $A$ and $B$ cells are distinct, but it is not easy to definitively allocate all cells to a specific hemilineage. The $12 \mathrm{~A}$ cells occupy more lateral positions (Fig 10e and g). The two hemilineages produce coherent and distinct primary neurite bundles into the neuropil. The 12A primary neurites enter the neuropil from a mid-ventral position and project dorsally (Fig 10e). Shortly after entering the neuropil the primary neurites split to produce three projections that parallel the prothoracic $12 \mathrm{~A}$ projections. The more lateral projection (aid) curves around the lateral edge of the tectulum to produce an arborization of varicose 
processes in dorsal-most tectulum largely restricted to the ipsilateral side except for a single projection that extends across the tectulum to the contralateral side (aid, Fig 10e). At the lateral edges of the projection, on both sides of the tectulum, the aid projection enters DLT and projects anteriorly to produce a small terminal arborization in the dorsal regions of the anterior tectulum (aida, Fig 10e, $f$ and $g$ ). There is no evidence of a branch that corresponds to the prothoracic aia branch but there is a projection that resembles ad which turns medially and, as it approaches the midline, projects dorsally into the tectulum where it produces an array of fine processes close to the aid arborizations (ad, Fig 10g and h). The ad branch also projects medially and ventrally to produce an arborization that spans the midline to produce an almost symmetrical projection that overlaps with the $12 \mathrm{~B}$ arborizations (aidv, Fig $10 \mathrm{~g}$ and h).

Like in the prothorax the $12 \mathrm{~B}$ primary projection enters the neuropil close to the midline and project dorsally before curving to cross the midline at the level of DLV and extend laterally to produce a complex arborization in contralateral leg neuropil, including the mechanosensory neuropil (Fig 10e). At its most distal point the projection splits (Fig $10 \mathrm{~g}$ ) to produce two arborizations. The first, continues ventrally to enter the mechanosensory neuropil to produce a diffuse varicose arborization in the mechanosensory and intermediate neuropil on both sides of the midline (bi, Fig 10h). The second projection curves laterally and dorsally (bii, Fig 10e, f and h) and into VLT to extend anteriorly and arborize in the posterior most prothoracic leg neuropil (bii, Fig 10e and f). There is also a small fine arborization ipsilateral to the cells that overlaps the $12 \mathrm{~A}$ arborization.

Metathorax - In the metathorax only $12 \mathrm{~B}$ survives and it is possible to see the characteristics of this hemilineage in isolation. The cells form a slightly "stretched' cluster on the posterior medial edge of the neuromere (Fig 10i) and produce a single primary projection that extends anteriorly to enter the neuropil at the anterior of the neuromere (Fig 10i) to cross the midline and recurve posteriorly and arborize in the intermediate and mechanosensory neuropil of the contralateral leg neuropil (Fig 10i and I). Close to the midline the neurons produce an array of fine processes that extend anteriorly into the ventral half of the posterior tectulum (Fig 10i and k). As in the mesothorax the distal end of the primary projection produces a dispersed array of varicose branches that ramify through 
the intermediate and mechanosensory neuropil (Fig 10I) as well as a projection that extends anteriorly in VTV to arborize in the mesothoracic ventral leg neuropil (arrows, Fig 10j).

\section{Lineage 16 (NB 1-1)}

Lineage 16 is found in all three thoracic neuromeres but only the B hemilineage survives in the adult. The anatomical organization of the lineage is largely the same in all three neuromeres (Fig 11a) producing a population of local interneurons that arborize in dorsal ipsilateral leg neuropil and tectulum.

The 16B cells form a tight cluster but their position varies by segment: in the prothorax they sit in the anterior medial of the ventral surface, in the mesothorax they sit more laterally, and in the metathorax they are displaced to a mid-neuromere position on the lateral edge of the neuromere (Fig 11a). In the prothorax, the cells produce a single primary projection that enters the neuropil at an anterior medial point and projects posteriorly before splitting to produce two distinct projections. One projection turns laterally (bl, Fig 11a and b) to produce a dense arborization in dorsal and lateral regions of anterior ipsilateral leg neuropil. The second projection turns medially to cross the midline and arborize both sides of the midline in the anteriormost tectulum ventral to $\mathrm{HT}$ and the cervical connectives and medial to DLV (bm, Fig 11a and b). In the mesothorax the same basic pattern is seen with a single primary neurite entering the neuropil bifurcating to produce an arborization in lateral leg neuropil (bl, Fig 11a \& c) and a medial projection that extends dorsally and anteriorly to arborize in the dorsal tectulum over T2 and contralaterally (bm, Fig 11a and c). In the metathorax the projection is comparable to other neuromeres (bl, Fig 11a, $d$ and e) but the medial projection is more extensive projecting dorsally and anteriorly to produce an arborization in the dorsal, posterior tectulum and extending anteriorly to overlap its mesothoracic homolog in mid tectulum (bm, Fig 11a and d).

\section{Lineage 17 (NB 2-5)}

Lineage 17 is found only in the meso and metathoracic neuromeres and only the $A$ hemilineage survives to the adult. We only generated usable MARCM clones for the mesothoracic neuromere. The mesothoracic $17 \mathrm{~A}$ cells sit as compact cluster on the dorsolateral surface at the anterior of the neuromere. The cells produce a short, robust 
primary neurite bundle that enters the neuropil in the dorsolateral corner of the neuromere. On entry to the neuropil the bundle splits to produce three projections, that exactly mirrors its undifferentiated form in the larva (Truman et al., 2004). The first projection (ad) projects dorsally in a posterior medial direction into the dorsal most ipsilateral tectulum (Fig $11 \mathrm{f}$ and g). In the tectulum the projection produces a three part arborization producing an arborization adjacent to the midline that extends anteriorly to terminate in dorsal tectulum (ada, Fig $11 \mathrm{f}$ and $\mathrm{h}$ ), a medially directed arbor that crosses the midline to terminate in the mid ventral tectulum (adc, Fig $11 \mathrm{f}$ and $\mathrm{h}$ ) and a posterior arborization (adp, Fig $11 f$ and $h$ ) that runs ventrally to terminate in the ventral tectulum adjacent to the midline.

The second projection (am, Fig 11f) projects medially and ventrally along the posterior edge of the ovoid before making and abrupt turn close to the midline to project posteriorly and laterally to arborize in lateral and ventral regions of the tectulum (am, Fig $11 \mathrm{f}$ and g). The third projection (av) projects ventrally and medially to produce a complex arborization in the primary mechanosensory (sn, Fig 11h) and intermediate neuropil.

\section{Lineage 19 (NB 6-2)}

Lineage 19 is found in all three thoracic neuromeres and both $A$ and $B$ hemilineages survive in all three thoracic neuromeres in the adult. The projections of all three are broadly the same in the thoracic neuromeres with some variations with the A hemilineage producing a population of local interneurons in ipsilateral leg neuropil and the B hemilineage producing complex intersegmental interneurons in the tectulum.

In the prothorax the $19 \mathrm{~A}$ and $\mathrm{B}$ cells sit at the dorsolateral edge at the posterior margin of the neuromere. The A and B cells can be resolved with the A cells ventral to B (Fig 12a). The 19A cells have a short primary neurite that enters the neuropil laterally (arrow, Fig 12a and b) that projects medially and dorsally to produce an arborization of fine branches that ramify through the dorsal and lateral regions of ipsilateral leg neuropil (Fig 12a and b) overlapping with 19B arborizations in the tectulum. The arborizations are restricted almost entirely to the hemineuromere of origin with only a single process crossing the midline. 
The prothoracic 19B hemilineage is very reduced ( $<10$ neurons) and produces a simple projection (Fig 12a). The 19B primary neurites enter the neuropil dorsal to 19A and projects directly at the same dorsoventral plane to cross the midline (Fig 12a and b). Ipsilaterally it produces an anteriorly directed projection that arborizes in anterior tectulum just medial to the HT (bi, Fig 12a and b). On the contralateral side the primary projection produces two anteriorly directed projections (bm and bl, Fig 12a and b). The medial projection (bm) produces a small arborization that is positioned similarly to the ipsilateral bi projection (Fig $12 \mathrm{a}$ and $\mathrm{b})$. The lateral projection enters the HT and projects anteriorly into the cervical connectives (bl, Fig 12a and b).

In the mesothorax the $19 \mathrm{~A}$ and $\mathrm{B}$ cells are completely separate, the $19 \mathrm{~A}$ somata form a diffuse cluster on dorsolateral edge of the neuromere and the 19B somata sit on the dorsal surface of the ganglion (Fig 12f). The 19A primary projection runs posteriorly between the cell cortex and neuropil before turning to enter the neuropil at the posterior lateral corner of the dorsal neuropil (arrow, Fig 12f) and projects medially and ventrally to arborize extensively in ipsilateral leg neuropil, with little or no evidence of contralateral or intersegmental projections (Fig $12 \mathrm{f}$ and $\mathrm{j}$ ).

The 19B cells produce a coherent primary projection that enters the neuropil from dorsal and produces an arcing projection the almost circumscribes the outer limits of the tectulum, crossing the midline and turning back to dorsal on the contralateral side (Fig $12 \mathrm{f}$ and j). In the course of its projection the primary projection produces 8 secondary projections. The first projection (bi) turns almost perpendicularly to enter the HT and project anteriorly through the mesothoracic neuromere to terminate anterior tectulum, producing diffuse dorsal arborizations in the lateral and ventral sections through the length of the tectulum (bi, Fig 12f-i). The second projection (bii) runs dorsally to produce a dense arborization in the posterior ipsilateral tectulum before extending posteriorly into the metathoracic leg neuropil (bii, Fig 12f, j and k). This projection partially overlaps the arborizations of the metathoracic 19B. Either side of the midline the primary projection produces a bilaterally symmetrical pair of dorsally directed secondary projections (19biii and 19biv) that extend dorsally and anteriorly curving laterally to produce dense arborizations in dorsal most regions of the tectulum (biii and biv, Fig $12 \mathrm{f}$ and i). Having crossed the midline, the primary projection extends laterally to the HT to project anteriorly and posteriorly. The anterior 
projection arises almost perpendicular to the primary projection (bv), mirroring the contralateral bi projection it extends anteriorly in $\mathrm{HT}$ through the mesothorax to terminate in dorsal anterior tectulum (bv, Fig 12f, g and h). The posterior projection (bvi) extends posteriorly and arborizes in the dorsal, posterior tectulum (bvi, Fig $12 f$ and k). There is a small but discrete projection that branches ventrally off the primary projection (bvii) and turns anteriorly to produce an extensive arborization in the ovoid (bvii, Fig 12h-j). The final projection (bviii) extends anteriorly in DLT to the prothoracic neuropil to arborize in dorsal leg neuropil (Fig 12f, g and j).

In the metathorax the $19 \mathrm{~A}$ and $\mathrm{B}$ cells are separate. The 19A somata sit on the dorsolateral edge at the posterior of the neuromere whereas the 19B cells sit on the dorsal surface at the anterior of the neuromere (Fig 12a). The 19A primary projections enter the metathoracic neuropil from a dorsolateral position and produces an arborization that ramifies throughout ipsilateral leg neuropil mainly in the medial and lateral neuropil (Fig 12a and e). The projection is local and remains within the hemineuromere of origin.

The 19B primary projections enter the neuropil from the dorsal surface and project ventrally and posteriorly before making and abrupt turn anteriorly and medially. As it runs anteriorly the projection produces four distinct branches. The first produces a compact but dense arborization (bi, Fig 12a, and c) that extends anteriorly to almost fill the posterior ipsilateral tectulum. The second projection (bii, Fig 12a, and d) produces a dense arborization in anterior metathoracic leg neuropil that overlaps the 19A arborization. The primary projection crosses the midline in the Haltere Commissure and splits to produce posteriorly (biii) and anteriorly (biv) directed projections. The biii projection curves laterally to produce a claw like projection of varicose arbors that mirror the ipsilateral bi projection (Fig 12a, $d$ and e). The biv projection enters HT and runs anteriorly through the meso and prothoracic neuromeres to enter the cervical connectives (biv, Fig 12a-c).

\section{Lineage 20/22 (NB 5-4/5-7)}

We have produced 19 MARCM clones that include the lineages produced by NBs 20 and 22 and like in the larva we have been unable to find any characteristics in their projections that discriminate the two NBs. We therefore deal with them as a single lineage with the same characteristics. The 20/22 lineages are found in all three thoracic neuromeres and only the A hemilineage survives in adults. The projections of the Lineage $20 / 22$ neurons are virtually 
identical in all three neuromeres producing a population of local interneurons restricted to the ipsilateral hemineuromere (Fig 13a-e). Occasionally the labelled clones included two motorneurons that are the remnants of the " $\mathrm{B}$ " portion of the lineage (arrows, Fig 13e).

The 20/22 cells sit on the posterior ventral region of the neuromere and produce primary neurites that project directly into the ipsilateral neuropil and arborize extensively within the ipsilateral leg neuropil (Fig 13a-e) and almost defines the limits of that neuropil. There are slight arborizations in the primary mechanosensory neuropils (sn, Fig 13b, c and e). In all three neuromeres there is also a dorsal projection that produces an arborization in ventral regions of the tectulum, ventral to MTD and ITD (Fig 13a-e) and restricted to the ipsilateral side.

\section{Lineage 21 (NB 4-3)}

Lineage 21 produces a population of local interneurons restricted to the ipsilateral hemineuromere. It is found in all 3 thoracic neuromeres but only the $21 \mathrm{~A}$ hemilineage survives to the adult stage. Although the $21 \mathrm{~A}$ projection appears different in the three thoracic neuromeres, it is essentially the same projection pattern relative to the axis of the leg neuropil (Fig 13f) 
In the prothorax the $21 \mathrm{~A}$ cells form a single cluster at the ventrolateral edge at the posterior of the neuromere (Fig 13f) whereas in the meso and metathorax the cells sit more medially (Fig 13f). In all three neuromeres the primary neurites form a single bundle that inserts into the ipsilateral neuropil and forms a very characteristic $\mathrm{T}$ shape as the primary projection splits to extend in opposite directions (Fig 13f). One branch extends laterally (al, Fig 13f) and the other medially (am, Fig 13f), with differences in orientation in each neuromere. The al projection extends laterally and ventrally to produce an extensive arborization in the leg neuropil, almost defining its extent with the exception of the primary mechanosensory neuropil (sn, Fig 13g, h, j and k). The am branch, by contrast, projects dorsally into ventral tectulum where it makes a second bifurcation with one branch crossing the midline (amm, Fig 13f) to form a small arborization in both ipsilateral and contralateral tectulum ventral to MTD and LTD (amm, Fig 13f, h-j). The second branch (ama, Fig 13f) extends anteriorly in the meso and metathorax but posteriorly in the prothorax to arborize in the ipsilateral ventral tectulum (Fig 13f, h, i-j) at the same dorso-ventral plane as the amm arborization. 


\section{Discussion}

In this study we have presented and analyzed the adult morphology of post-embryonically induced MARCM clones to reveal the anatomy of the vast majority of adult neuronal lineages in the thoracic neuromeres of the VNS. This work builds on previous studies of the developmental origins of the adult neurons of the VNS (Harris et al., 2015; Shepherd et al., 2016; Truman et al., 2004) to produce a definitive description of the clonal organization of neurons in the adult VNS. We present data for 58 of the 66 postembryonic thoracic lineages (85 of the 98 possible thoracic hemilineages), excluding the motor neuron producing lineages (15 and 24 found in all thoracic hemineuromeres). The data are summarized schematically in figure 14. Although we do not provide full coverage of the adult lineages, we have at least one representative of every post embryonic NB with the exception of lineage 18 which remained resistant to MARCM analysis. The information we present provides a fundamental baseline of understanding of the functional organization of the adult VNS. By understanding the morphological features of these neurons, we can begin to define and test the rules by which neuronal circuits are assembled during development and to understand the functional logic and evolution of neuronal networks. As demonstrated with the vertebrate spinal cord, knowledge of the developmental origins of neurons particularly the cell type specific expression of 'developmental' transcription factor has been incredibly useful for understanding the functional organization of the complex sensory motor systems generating locomotor behavior (Grillner and Jessell, 2009). Drosophila is an equally well-understood model for developmental biology, with powerful genetics and a sophisticated array of locomotor behaviors controlled by a relatively small nervous system. Detailed understanding of the developmental rules and organization of the Drosophila VNS is therefore an important step to establishing the adult VNS as a model system to understand how complex arrays of neurons generate behaviors.

\section{Hemilineages are functional units}

By its nature the MARCM technique reveals whole lineages, it is, however, the hemilineages that represent the functional units of the VNS (Harris et al., 2015). It is therefore important to understand the organisation of the hemilineages within each adult lineage and we have sought to unravel the hemilineages from the full lineage structure. In the VNS, the A and B 
hemilineages have distinct anatomies and functions, so that even in cases in which both the $A$ and $B$ hemilineages survive [e.g. lineages $19,12,13,6,1,38,11$ ], the hemilineages frequently have limited overlap so it is possible to discriminate the anatomy of the $A$ and $B$ hemilineages in a clone. Furthermore, based on their anatomical organization within the VNS neuropil it is possible to infer the functional roles of each hemilineage based on the neuropil regions into which their axonal and dendritic processes extend. The presumed functional roles of each hemilineage, based on morphology, are summarised in fig 14.

\section{Ventrally arborizing hemilineages associated with leg control}

Of the 33 hemilineages in a hemineuromere revealed in this study, 15 (including the motor hemilineages $15 \mathrm{~B}$ and $24 \mathrm{~B}$ ) are associated with the leg neuropils and can be considered as part of the circuitry controlling leg movements. Interestingly all of the leg specific hemilineages have common features. They are invariably based on local interneurons with arborizations restricted to a single hemineuromere and show very little intersegmental variation. Furthermore, each leg hemilineage appears to be composed of a homogeneous neuron types with all members of the hemilineage showing the same basic arborizations. In most cases the arborizations span the leg neuropil from the ventral primary sensory input regions to more dorsal and lateral regions of neuropil that contain the dendrites of leg motorneurons. It is, therefore, likely that all of these hemilineages play a role in sensorymotor processing for the leg. Each hemilineage, though, has its own unique morphology that suggests specific roles in leg control. Some hemilineages have dendritic processes restricted to specific sensory domains suggesting involvement in the processing of specific sensory modalities. For example, hemilineage $10 B$ has dendrites tightly restricted to mVAC and intermediate neuropil indicative of being involved in processing of sensory information from the "club" type sensory afferents from the femoral chordotonal organ (FCO)(Mamiya, Gunung, \& Tuthill, unpublished) and from campaniform sensilla on the legs. The $10 B$ neurons also have intersegmental projections that overlap with the dendrites of their segmental homologs suggesting a role in coordinating sensory inputs between different legs. In another examples the dendrites of 23B neurons are restricted to the primary mechanosensory neuropil indicative of involvement in processing information from leg exteroceptors. Of all the leg specific hemilineages, the 1B neurons have the most restricted 
arborization which is limited to the ventral sensory domains such as mVAC and the primary mechanosensory neuropils suggesting that these neurones are likely to pay a role in modulating sensory inputs to other leg interneurons.

Leg hemilineages 4B, 13A and B, 14B and 19A have more complex and dispersed arborizations with widespread distribution through the leg neuropil. These hemilineages are still local interneurons but are likely to be involved in more complex sensory motor processing. This can be exemplified by the 13B and 14A interneurons both of which resemble the populations of midline spiking interneurons that process information from the somatosensory map to the myotopic motor map (Malcolm Burrows, 1996; M. Burrows \& Newland, 1993, 1994). Both hemilineage 13B and 14A neurons in flies are anatomically similar to their grasshopper homologs and the fly neurons and both are likely to be involved in maintaining the integrity of spatial information from sensory inputs (Murphey et al., 1989a and b) to motor outputs (Brierley et al., 2012) of the leg.

Whilst the leg hemilineages are relatively homogenous populations of neurons, some of the leg hemilineages clearly contain different neuron subtypes. This is best seen in lineage 9A which is likely to have at least three different subtypes, each serving slightly different role in sensory processing, with one type targeting mVAC and "club" type FCO sensory afferents, a second targeting intermediate neuropil and the "claw" type sensory afferents from the FCO and a third in ventral-most medial sensory neuropil which based on its anatomy is likely to process inputs from chemosensory neurons. Further studies to tease apart the structure and functions of these sub lineages will provide important insight into cellular diversity of the adult VNS.

\section{Dorsally arborizing hemilineages associated with wing control}

Whilst many thoracic hemilineages have major arborizations in the dorsal flight neuropil surprisingly only 7 hemilineages (Fig 14) have arborizations that are exclusively restricted to dorsal neuropil. Even these hemilineages have some arborization, albeit in some case very small, that impinge on the leg neuropils. The best example of a wing specific hemilineage is $6 A$ and $B$, which produces a dense arborization in the dorsal most regions of the tectulum, 
but few projections outside the tectulum. The other hemilineages that can be considered to be primarily dedicated to wing neuropils are 2A, 3B, 11B, 12A and 19B (Fig 14).

Of the remaining hemilineages with arborizations in the dorsal neuropils ( $n=9$, Fig 14) they all extend significant arborizations into leg neuropils and clearly span both compartments indicating that they play a key role in the coordination of leg and wing activity. This observation that there are very few hemilineages appear to be exclusively restricted to the dorsal flight neuropils correlates well with the behavioural work done by Harris et al (2015) who using TrpA activation of hemilineages also found that few hemilineages that when stimulated produced only wing related behaviours. It is interesting to speculate whether the relatively small number of exclusively flight related hemilineages is a reflection of the apterygote origins of insects.

In some of these hemilineages the major component of the projection is based in the wing neuropils with only a minor component in the leg neuropils (e.g. 6B, 7B, 8B, 12A and 17A) but there are others where the majority of the arborizations are in leg neuropils (e.g. 1A, $12 B, 16 B, 20 / 22 A$ and $21 A$ ) possibly reflecting the polarity of information flow between leg and wing neuropils in each hemilineage. For instance, the mesothoracic hemilineage 7A has complex projections throughout the dorsal and ventral tectulum with most of the ipsilateral arborization being dendritic with output arborizations in both ipsilateral and contralateral tectulum. The most striking feature of 7A, however, are the axonal projections into all three contralateral leg neuropils and the large diameter projection to the lateral regions of the ipsilateral mesothoracic neuromere. Whatever the full function of 7A neurons, in this neuromere a key part of that output is to make significant outputs to leg motor neurons. On the other hand, hemilineage 16B has its major arborizations in the ipsilateral leg neuropil but it also has a clear projection into the ipsilateral tectulum transferring information from the leg neuropil to wing/haltere circuits.

In contrast to the leg hemilineages, the dorsally projecting hemilineages are also more diverse in their structure. The hemilineages that converge on the tectulum are often intersegmental and span left and right hemineuromeres. There is also considerable segmental variation and specialisation with a general convergence of arborizations on the 
mesothoracic regions of the tectulum and the reduced complexity or absence of hemilineages in the prothorax (17A and 19B) or metathorax (3B and 12A). There is also greater heterogeneity of cell types within the dorsally projecting hemilineages, the most notable examples being hemilineages $8 \mathrm{~B}$ and $19 \mathrm{~B}$. In both case there are multiple projections targeting specific regions of the tectulum. In hemilineage $8 \mathrm{~B}$ there are 6 projections some ipsilateral and others contralateral that both ascend and descend in different tracts. Given that there are no known neurons that share all of these projections it is likely that different classes of neurons are responsible for the different projections. Likewise, hemilineage 19B which has multiple ascending and descending projections, is likely to be made up of different neuronal types.

Not all of the dorsally projecting hemilineages show such heterogeneity, there are examples that appear to be composed of a small number of types $(1 A, 2 A, 6 A$ and $B, 12 A$ and $B, 16 B$, $20 / 22 \mathrm{~A}$ and $21 \mathrm{~A}$ ). This is best seen with hemilineages $20 / 22 \mathrm{~A}$ and $21 \mathrm{~A}$ which have all of the features of leg specific hemilineages of local interneurons with similar morphology and little segmental variation or diversity.

The thoracic hemilineages vary in the heterogeneity of neurons they contain. This undoubtedly reflects the different functional requirements of the control circuitry with the more homogenous hemilineages being collections of parallel components, that provide parallel processing channels to control complex sensory to motor integration. The hemilineages with greater heterogeneity are more likely have more diversified functions and behavioral modules.

The demonstration that some hemilineages are more heterogeneous in terms of the neuron types they produce has implications for breaking the organization VNS circuits into behavioural components indicating that there may be some levels of complexity that might resist simple analysis. That consideration aside, although there is heterogeneity it is not vast, in the most extreme examples (e.g. mesothoracic 19B) there is likely to be a relatively small number $(<10)$ of different neuron types. Thus, it should still possible to caricature the hemilineages as populations of neurons that share common anatomical and physiological characteristics serving discrete behavioural repertoires. 


\section{Completeness of the lineages}

In presenting these lineages we selected the most complete examples as determined by the extent of the arborizations and the number of cells associated with each clone. Where possible we selected examples with the largest number of cells and the most extensive arborizations. All of the clones were induced by heat shocking first instar larvae and timed to ensure we reveal the full complement of the postembryonic lineages. We cannot be absolutely certain we have not missed some early born progeny but in examples where we know the identities of the earliest born postembryonic neurons (e.g. 14B and 23B) we can confirm those neurons are present in our clones. Furthermore, the primary projections of the adult lineages can be related to the projections described for the postembryonic lineages in the late larval stages by Truman et al. (Truman et al., 2004) and we see no missing projections. We are therefore confident that the clones presented reflect the full adult specific lineages.

\section{Methods}

\section{Generation of MARCM clones}

The MARCM technique was used, in which the FLP/ FRT system induced clones that lacked GAL80, a suppressor of GAL4, to make CD8::GFP-labeled clones in an unlabeled background (Lee and Luo, 2001). The GAL4 drivers used for MARCM were elav C155 and OK371. Eggs were collected on apple juice plates for 2 hours, held for 24 hours (both at $25^{\circ} \mathrm{C}$ ), and the larvae were heat shocked between 3 and 5 hours after hatching. Larvae were reared on standard food at $25^{\circ} \mathrm{C}$. Nervous systems were dissected from 1-day-old adults.

\section{Preparation and examination of tissues}

Dilutions of the antibodies used in immunostaining are given below and in Table 1. Tissues were dissected in PBS (phosphate-buffered saline, pH7.8) and fixed in 4\% buffered formaldehyde for 1 hour at room temperature. Fixed tissues were washed in PBSTX (PBS with 1\% Triton X-100, Sigma, St. Louis, MO), incubated in 10\% normal donkey serum (Jackson ImmunoResearch, West Grove, PA; Cat. no. 017-000-001 RRID:AB_2337254) for up to 6 hours, and then in 1:1,000 rabbit anti-GFP (Molecular Probes (Invitrogen), Eugene, OR; 
Cat. no. A11122 RRID:AB_221569), 1:40 rat anti-N-cadherin (Developmental Studies Hybridoma Bank, lowa City, IA; Cat. no. DN-Ex 8 RRID:AB_528121), and 1:40 mouse antineuroglian (Developmental Studies Hybridoma Bank; Cat. no. BP 104 anti-neuroglian RRID:AB_528402) overnight at $4^{\circ} \mathrm{C}$. Tissues were washed in PBS-TX and incubated with 1:500 AlexaFluor 488-conjugated donkey antirabbit (Jackson ImmunoResearch; Cat. no. 715545-151 RRID:AB_2341099), AlexaFluor 594-conjugated donkey antimouse (Jackson ImmunoResearch; Cat. no. 715- 585-151 RRID:AB_2340855), and AlexaFluor 647conjugated donkey antirat (Jackson ImmunoResearch; Cat. no. 712-605-153 RRID:AB_2340694) overnight at $4^{\circ} \mathrm{C}$. This is a standard antibody protocol we use for all our studies. The Neuroglian staining reveals the primary neurite scaffold of the hemilineages but the information provided by this channel was not shown in this study. Tissues were washed in PBS-TX, mounted onto poly-lysine-coated coverslips, dehydrated through an ethanol series, cleared in xylene, and mounted in DPX mountant (Sigma-Aldrich). Nervous systems were imaged on a Zeiss 710 confocal microscope. LSM files were contrast-enhanced as necessary. Z-projected images were created using ImageJ (http://rsbweb.nih.gov/ij/) RRID:SCR_001935, transverse sections were created with Vaa3D (Peng, et al. 2010) (http://www.vaa3d.org) RRID:SCR_002609 and figure montages made in Adobe Photoshop (San Jose, CA) (https://www.adobe.com/products/photoshop.html) , RRID:SCR_014199

\section{Image Processing}

Confocal image stacks of MARCM clones were aligned to standardized brain template based on the N-Cadherin expression pattern in the VNS of a female CantonS fly, available at the Virtual Fly Brain ( http://www.virtualflybrain.org), RRID:SCR_004229). The alignment process was adapted from (Court, 2017). Briefly: confocal VNC stacks were first converted to an 8-bit nrrd file format, pre-processed using the $\mathrm{N}$-cadherin channel to normalize contrast across samples, rotated to orient the VNS and the reference channel aligned to the template by nonrigid warping (Rohlfing \& Maurer, 2003) using the Computational Morphometry Toolkit (http://neuro.debian.net/pkgs/cmtk.html

) RRID:SCR_002234, as described in detail in (Jefferis et al., 2007). The signal channel containing the GFP expression pattern was then transformed using the warped mesh determined above, the two individual image files were then combined as separate channels 
and converted back to TIFF file format. The image files presented in this paper will be made available on Virtual Fly Brain ( http://www.virtualflybrain.org), RRID:SCR_004229

Antibody characterization

Anti-GFP (RRID:AB_221569) is a commercially available polyclonal anti-body raised in rabbit to purified GFP. In our studies its specificity is validated by internal controls such that the pattern of immunostaining consistently and precisely matches the patterns of fluorescence produced by Gal4-driven GFP expression in a vast array of different GAL4 lines including negligible immunoreactivity in non-GFP-expressing tissues. It has also been widely used in many other systems and organisms to successfully demonstrate the localization of expressed GFP.

Anti-neuroglian (RRID:AB_528402) is an IgG1 anti- body raised in mouse against a nervous system-specific 180 kD splice variant of Drosophila neuroglian ((Hortsch et al., 1990). The $180 \mathrm{kD}$ isoform can be purified to homogeneity and the derived amino acid sequence was identical to the sequence for the amino terminus of the 167 kD isoform ((Bieber et al., 1989; Hortsch et al., 1990). The antibody recognizes an epitope on the cytoplasmic segment of the long form of the protein (Hortsch et al., 1990). Null mutations in the nrg gene are lethal but hypomorphic mutations have greatly reduced expression (Hall \& Bieber, 1997). Neuroglian protein expression assessed using BP-104 in $\mathrm{nrg}^{3}$ mutants, which are temperature-sensitive, shows that at the restrictive temperature, labeling in neuroglian-positive neuronal processes was eliminated (Hall and Bieber, 1997).

The $n$-cadherin DN-Ex \#8 antibody (RRID:AB_528121) was raised by Iwai et al (1997) to the extracellular domain of Drosophila N-cadherin. Full validation of its specificity was provided in the original publication. In this study we do not use the antibody to report on any specific role of $\mathrm{N}$-Cadherin and use it as a counterstain to neuropil which reveals features related to the density of synaptic connections thereby enlacing the contrast of the VNS neuropil. 


\section{References}

Baek, M., \& Mann, R. S. (2009). Lineage and birth date specify motor neuron targeting and dendritic architecture in adult Drosophila. J Neurosci, 29(21), 6904-6916. doi:10.1523/JNEUROSCI.1585-09.2009

Bieber, A. J., Snow, P. M., Hortsch, M., Patel, N. H., Jacobs, J. R., Traquina, Z. R., . . Goodman, C. S. (1989). Drosophila neuroglian: a member of the immunoglobulin superfamily with extensive homology to the vertebrate neural adhesion molecule L1. Cell, 59(3), 447-460.

Birkholz, O., Rickert, C., Nowak, J., Coban, I. C., \& Technau, G. M. (2015). Bridging the gap between postembryonic cell lineages and identified embryonic neuroblasts in the ventral nerve cord of Drosophila melanogaster. Biol Open. doi:10.1242/bio.201411072

Brierley, D. J., Rathore, K., VijayRaghavan, K., \& Williams, D. W. (2012). Developmental origins and architecture of Drosophila leg motoneurons. J Comp Neurol, 520(8), 1629-1649. doi:10.1002/cne.23003

Brown, H. L., \& Truman, J. W. (2009). Fine-tuning of secondary arbor development: the effects of the ecdysone receptor on the adult neuronal lineages of the Drosophila thoracic CNS. Development, 136(19), 3247-3256. doi:10.1242/dev.039859

Burrows, M. (1996). The neurobiology of an insect brain: Oxford University Press.

Burrows, M., \& Newland, P. L. (1993). Correlation between the receptive fields of locust interneurons, their dendritic morphology, and the central projections of mechanosensory neurons. J Comp Neurol, 329(3), 412-426. doi:10.1002/cne.903290311

Burrows, M., \& Newland, P. L. (1994). Convergence of mechanosensory afferents from different classes of exteroceptors onto spiking local interneurons in the locust. $J$ Neurosci, 14(5 Pt 2), 3341-3350.

Büschges, A., Akay, T., Gabriel, J. P., \& Schmidt, J. (2008). Organizing network action for locomotion: insights from studying insect walking. Brain Res Rev, 57(1), 162-171. doi:10.1016/j.brainresrev.2007.06.028

Court, R. (2017). Dissecting the ventral nervous system in Drosophila melanogaster. In. PhD Thesis, University of Edinburgh.

Enriquez, J., Rio, L. Q., Blazeski, R., Bellemin, S., Godement, P., Mason, C., \& Mann, R. S. (2018). Differing Strategies Despite Shared Lineages of Motor Neurons and Glia to Achieve Robust Development of an Adult Neuropil in Drosophila. Neuron, 97(3), 538554.e535. doi:10.1016/j.neuron.2018.01.007

Enriquez, J., Venkatasubramanian, L., Baek, M., Peterson, M., Aghayeva, U., \& Mann, R. S. (2015). Specification of individual adult motor neuron morphologies by combinatorial transcription factor codes. Neuron, 86(4), 955-970. doi:10.1016/j.neuron.2015.04.011

Grillner, S., \& Jessell, T. M. (2009). Measured motion: searching for simplicity in spinal locomotor networks. Curr Opin Neurobiol, 19(6), 572-586. doi:10.1016/j.conb.2009.10.011

Hall, S. G., \& Bieber, A. J. (1997). Mutations in the Drosophila neuroglian cell adhesion molecule affect motor neuron pathfinding and peripheral nervous system patterning. J Neurobiol, 32(3), 325-340. 
Harris, R. M., Pfeiffer, B. D., Rubin, G. M., \& Truman, J. W. (2015). Neuron hemilineages provide the functional ground plan for the Drosophila ventral nervous system. Elife, 4. doi:10.7554/eLife.04493

Hortsch, M., Bieber, A. J., Patel, N. H., \& Goodman, C. S. (1990). Differential splicing generates a nervous system-specific form of Drosophila neuroglian. Neuron, 4(5), 697-709.

Iwai, Y., Usui, T., Hirano, S., Steward, R., Takeichi, M., \& Uemura, T. (1997). Axon patterning requires DN-cadherin, a novel neuronal adhesion receptor, in the Drosophila embryonic CNS. Neuron, 19(1), 77-89.

Jefferis, G. S., Potter, C. J., Chan, A. M., Marin, E. C., Rohlfing, T., Maurer, C. R., \& Luo, L. (2007). Comprehensive maps of Drosophila higher olfactory centers: spatially segregated fruit and pheromone representation. Cell, 128(6), 1187-1203. doi:10.1016/j.cell.2007.01.040

Jia, X. X., \& Siegler, M. V. (2002). Midline lineages in grasshopper produce neuronal siblings with asymmetric expression of Engrailed. Development, 129(22), 5181-5193.

Lacin, H., \& Truman, J. W. (2016). Lineage mapping identifies molecular and architectural similarities between the larval and adult Drosophila central nervous system. Elife, 5, e13399. doi:10.7554/elife.13399

Lacin, H., Zhu, Y., Wilson, B. A., \& Skeath, J. B. (2014). Transcription factor expression uniquely identifies most postembryonic neuronal lineages in the Drosophila thoracic

Lee, T., \& Luo, L. (2001). System. Development, 141(5) 1011-1021. doi:101242/dev. 102178 Drosophila neural development. Trends Neurosci, 24(5), 251-254.

Lupo, G., Harris, W. A., \& Lewis, K. E. (2006). Mechanisms of ventral patterning in the vertebrate nervous system. Nat Rev Neurosci, 7(2), 103-114. doi:10.1038/nrn1843

Mamiya, A., Gunung, P., \& Tuthill, J. (unpublished). Neural coding of leg proprioception in Drosophila. In. doi: https://doi.org/10.1101/274498.

Merritt, D. J., \& Murphey, R. K. (1992). Projections of leg proprioceptors within the CNS of the fly Phormia in relation to the generalized insect ganglion. J Comp Neurol, 322(1), 16-34. doi:10.1002/cne.903220103

Murphey, R. K., Possidente, D., Pollack, G., \& Merritt, D. J. (1989). Modality-specific axonal projections in the CNS of the flies Phormia and Drosophila. J Comp Neurol, 290(2), 185-200. doi:10.1002/cne.902900203

Murphey, R. K., Possidente, D. R., Vandervorst, P., \& Ghysen, A. (1989). Compartments and the topography of leg afferent projections in Drosophila. J Neurosci, 9(9), 3209-3217.

Namiki, S., Dickinson, M. H., Wong, A. M., Korff, W., \& Card, G. M. (2018). The functional organization of descending sensory-motor pathways in. Elife, 7. doi:10.7554/eLife.34272

Peng, H., Ruan, Z., Long, F., Simpson, J. H., \& Myers, E. W. (2010). V3D enables real-time 3D visualization and quantitative analysis of large-scale biological image data sets. Nat Biotechnol, 28(4), 348-353. doi:10.1038/nbt.1612

Power, M. E. (1948). The thoracico-abdominal nervous system of an adult insect, Drosophila melanogaster. J Comp Neurol, 88(3), 347-409.

Robertson, R. M., Pearson, K. G., \& Reichert, H. (1982). Flight interneurons in the locust and the origin of insect wings. Science, $217(4555), 177-179$.

doi:10.1126/science.217.4555.177 
Rohlfing, T., \& Maurer, C. R. (2003). Nonrigid image registration in shared-memory multiprocessor environments with application to brains, breasts, and bees. IEEE Trans Inf Technol Biomed, 7(1), 16-25.

Schmid, A., Chiba, A., \& Doe, C. Q. (1999). Clonal analysis of Drosophila embryonic neuroblasts: neural cell types, axon projections and muscle targets. Development, 126(21), 4653-4689.

Shepherd, D., Harris, R., Williams, D. W., \& Truman, J. W. (2016). Postembryonic lineages of the Drosophila ventral nervous system: Neuroglian expression reveals the adult hemilineage associated fiber tracts in the adult thoracic neuromeres. J Comp Neurol, 524(13), 2677-2695. doi:10.1002/cne.23988

Shepherd, D., \& Laurent, G. (1992). Embryonic development of a population of spiking local interneurones in the locust (Schistocerca gregaria). J Comp Neurol, 319(3), 438-453. doi:10.1002/cne.903190309

Siegler, M. V., Pankhaniya, R. R., \& Jia, X. X. (2001). Pattern of expression of engrailed in relation to gamma-aminobutyric acid immunoreactivity in the central nervous system of the adult grasshopper. J Comp Neurol, 440(1), 85-96.

Strausfeld, N. J., \& Seyan, H. S. (1985). Convergence of visual, haltere, and prosternal inputs at neck motor neurons of Calliphora erythrocephala. Cell and Tissue Research, 240, 601-615.

Thompson, K. J., \& Siegler, M. V. S. (1991). Anatomy and physiology of spiking local and intersegmental interneurons in the median neuroblast lineage of the grasshopper. In (Vol. 305, pp. 659-675). Journal of Comparative Neurology.

Thor, S., \& Thomas, J. B. (1997). The Drosophila islet gene governs axon pathfinding and neurotransmitter identity. Neuron, 18(3), 397-409.

Truman, J. W., \& Bate, M. (1988). Spatial and temporal patterns of neurogenesis in the central nervous system of Drosophila melanogaster. Dev Biol, 125(1), 145-157.

Truman, J. W., Moats, W., Altman, J., Marin, E. C., \& Williams, D. W. (2010). Role of Notch signaling in establishing the hemilineages of secondary neurons in Drosophila melanogaster. Development, 137(1), 53-61. doi:10.1242/dev.041749

Truman, J. W., Schuppe, H., Shepherd, D., \& Williams, D. W. (2004). Developmental architecture of adult-specific lineages in the ventral CNS of Drosophila. Development, 131(20), 5167-5184. doi:10.1242/dev.01371

Witten, J. L., \& Truman, J. W. (1998). Distribution of GABA-like immunoreactive neurons in insects suggests lineage homology. J Comp Neurol, 398(4), 515-528. 


\section{Figure legends}

Figure 1 Schematic presentation of a transverse section through the mesothoracic neuromeres illustrating the major neuropil regions and longitudinal tracts and their abbreviations. After Merritt and Murphey (1992).

Figure 2 MARCM clones for Lineages 4 and 13. (a-g) Lineage 4 (a) Maximum Z projection of the entire VNS showing the full projection of the prothoracic lineage 4 (green). (b-c) composite of 10 horizontal $Z$ stacks though the ipsilateral prothoracic neuromere selected to reveal details of the lineage. (d) Maximum Z projection of the entire VNS showing the full projection of the metathoracic lineage 4 (green). (e-f) Transverse sections through the specimen shown in (a) the plane of the sections is indicated by the brackets in (a). (g) Transverse section through the specimen shown in (d), the plane of the section is indicated by the bracket in panel D. (h-p) Lineage 13 (h-i) Prothorax - (h-i) composite of 10 horizontal $Z$ stacks taken at different dorsal-ventral planes though the prothoracic neuromere selected to reveal details of the lineage, the plane of the sections is indicated by the brackets in (j). (j) Transverse section through the specimen in (h-i) the plane of the section is indicated by the brackets in (i). (k-m) Mesothorax - (k-l) composite of 10 horizontal Z stacks taken at different dorsal-ventral planes though the mesothoracic neuromere selected to reveal details of the lineage, the plane of the sections is indicated by the brackets in $(\mathrm{m})$. ( $\mathrm{I}$ ) Transverse section through the specimen in $(k-m)$ the plane of the section is indicated by the brackets in (I). (n-p) Metathorax - (n-o) composite of 10 horizontal Z stacks taken at different dorsal-ventral planes though the metathoracic neuromere selected to reveal details of the lineage, the plane of the sections is indicated by the brackets in (o). (o) Transverse section through the specimen in (n-o) the plane of the section is indicated by the brackets in (o). Dotted lines outline neuropil regions relevant to the projections (sn -primary mechanosensory neuropil, $\mathrm{mVAC}$, tectulum and $\mathrm{Abd}=$ abdominal neuromeres. Key tracts and details of the projections are also indicated. Scale $=100 \mu \mathrm{m}$.

Figure 3 MARCM clones for Lineages $9,10,14$ and 23. (a-i) Lineage 9 (a) Maximum Z projection of the entire VNS showing the full projection of lineage 9 in all three thoracic neuromeres (T1 green, T2 Cyan and T3 magenta). (b-c) composite of 10 horizontal Z stacks though the ipsilateral prothoracic neuromere selected to reveal details of the lineage. (d-f) Transverse sections through lineages shown in (a), (d) Prothorax, (e) Mesothorax and (f) Metathorax. The plane of the sections is indicated by brackets in panel a. (g-i) Maximum $Z$ projections of OK371 MARCM clones of lineage 9 during pupal stages (green) that selectively reveals the contralateral component of lineage 9. (g) Prothorax in a pupal VNS at $24 \mathrm{~h}$ after pupation, (h) Mesothorax in a pupal VNS at $48 \mathrm{~h}$ after pupation, (j) Metathorax in a pupal VNS at $48 \mathrm{~h}$ after pupation. (j-n) Lineage 10 Maximum Z projection of the VNS showing the full projection of lineage 10 in pro and mesothoracic neuromeres (T1 green, T2 Cyan). $(k-n)$ Transverse sections through the preparations shown in (j), the plane of the sections is indicated by brackets in panel j. (p-s) Lineage 23 (p) Maximum Z projection of the VNS showing the full projection of lineage 23 in pro and mesothoracic neuromeres (T1 green, T2 Cyan). (q-s) Transverse sections through the preparations shown in (p), the plane of the sections is indicated by brackets in panel p. (t-w) Lineage $14(t)$ Maximum Z projection of the VNS showing the full projection of lineage 14 in all three thoracic neuromeres (T1 green, 
T2 Cyan, T3 Magenta). ( $u-w)$ Transverse sections through the preparations shown in (t), the plane of the sections is indicated by brackets in panel t. Dotted lines outline neuropil regions relevant to the projections (sn - primary mechanosensory neuropil, tectulum and $A b d=$ abdominal neuromeres. Key tracts and details of the projections are also indicated. Scale $=100 \mu \mathrm{m}$.

Figure 4 MARCM clones for Lineages 2 and 11. (a-i) Lineage 2 (a-c) Maximum Z projection of the VNS showing the full projection of lineage 2 (green) in all three thoracic neuromeres (a- T1, b- T2 and c- T3). (d-h) Transverse sections through lineages shown in (a-c), The plane of the sections is indicated by brackets in panels a-c. The example in Panels $\mathrm{c}, \mathrm{h}$ and $\mathrm{i}$ includes a single lineage 0 neuron clone (arrows). ( $j-1)$ Lineage 11 (j) Maximum Z projection of the VNS showing the full projection of lineage 11 (green) in the mesothoracic neuromere. (k-I) Transverse sections through lineage 11 shown in (j), The plane of the sections is indicated by brackets in panel $\mathrm{j}$. Dotted lines outline neuropil regions relevant to the projections (sn - primary mechanosensory neuropil, ovoid, and tectulum. Key tracts and details of the projections are also indicated. Scale $=100 \mu \mathrm{m}$.

Figure 5 MARCM clones for Lineage 6. (a-f) Prothoracic lineage 6 (a) Maximum Z projection of the VNS showing the full projection of the prothoracic lineage 6 (green). (b-f) Transverse sections through lineage 6 shown in (a), the plane of the sections is indicated by brackets in panel a. (g-k) Mesothoracic lineage 6 (g) Maximum Z projection of the VNS showing the full projection of the mesothoracic lineage 6 (green). (h-k) Transverse sections through lineage 6 shown in (g), The plane of the sections is indicated by brackets in panels g. (I-n)

Metathoracic lineage 6 (I) Maximum Z projection of the VNS showing the full projection of the metathoracic lineage 6 (green). (m-n) Transverse sections through lineage 6 shown in (I), The plane of the sections is indicated by brackets in panels I. Dotted lines outline neuropil regions relevant to the projections (sn - primary mechanosensory neuropil, ovoid, and tectulum. Key tracts and details of the projections are also indicated. Scale $=100 \mu \mathrm{m}$.

Figure 6 MARCM clones for Lineages 0 and 1. (a-i) Lineage 0 (a-c) Prothoracic lineage 0. (a) Maximum $Z$ projection of the VNS showing the full projection of the prothoracic lineage 0 (green). (b-c) Transverse sections through lineage 0 shown in (a), the plane of the sections is indicated by brackets in panel a. (d-f) Mesothoracic lineage $\mathbf{0}$. (d) Maximum Z projection of the VNS showing the full projection of the mesothoracic lineage 0 (green). (e-f) Transverse sections through lineage 0 shown in (d), the plane of the sections is indicated by brackets in panel D. (g-i) Metathoracic lineage $\mathbf{0}$. (g) Maximum Z projection of the VNS showing the full projection of the metathoracic lineage 0 (green). ( $h-i)$ Transverse sections through lineage 0 shown in (g), the plane of the sections is indicated by brackets in panel $g$. (j-i) Lineage $1 \mathbf{A}(\mathbf{j})$ Maximum $Z$ projection of the entire VNS showing the full projection of lineage $1 \mathrm{~A}$ in all three thoracic neuromeres (T1 green, T2 Cyan and T3 magenta). (k-r) Transverse sections through lineages shown in (j). The plane of the sections is indicated by brackets in panel $\mathrm{j}$. (p-r) Lineage 1B (p) Maximum Z projection of the VNS showing the full projection of the meso and metathoracic lineage 1Bs (T2 Cyan and T3 magenta). (q-r) Transverse sections through lineages in ( $p)$, the plane of the sections is indicated by brackets in panelp. Dotted lines outline neuropil regions relevant to the projections (sn - primary mechanosensory neuropil, mVAC, ovoid, tectulum and Abdominal neuromeres (Abd). Key tracts and details of the projections are also indicated. Scale $=100 \mu \mathrm{m}$. 
Figure 7 MARCM clones for Lineages 3 and 5. (a-h) Lineage 3 (a-c) Prothoracic lineage 3. (a) Maximum Z projection of the VNS showing the full projection of the prothoracic lineage 3 (green). (b-c) Transverse sections through lineage 3 shown in (a), the plane of the sections is indicated by brackets in panel a. (d-e) Mesothoracic lineage 3. (d) Maximum Z projection of the VNS showing the full projection of the mesothoracic lineage 3 (green). (e) Transverse sections through lineage 3 shown in (d), the plane of the sections is indicated by brackets in panel d. (f-h) Metathoracic lineage 3. (f) Maximum Z projection of the VNS showing the full projection of the metathoracic lineage 3 (green). (g-h) Transverse sections through lineage 3 shown in (f), the plane of the sections is indicated by brackets in panel $f$. The inset in $(f)$ is a composite of 10 horizontal $Z$ stacks though the ipsilateral metathoracic neuromere selected to reveal the characteristic shape of the primary projection. (i-m) Lineage 5 (I) Maximum Z projection of the VNS showing the full projection of the prothoracic lineage 5 (green). (j-m) Transverse sections through lineage 5 shown in (i), the plane of the sections is indicated by brackets in panel i. Dotted lines outline neuropil regions relevant to the projections (sn primary mechanosensory neuropil, mVAC, ovoid, tectulum and Abdominal neuromeres (Abd). Key tracts and details of the projections are also indicated. Scale $=100 \mu \mathrm{m}$.

Figure 8 MARCM clones for Lineage 7. (a-e) Mesothoracic lineage 7 (a) Maximum Z projection of the VNS showing the full projection of the mesothoracic lineage 7 (green). (bc) Transverse sections through lineage 7 shown in (a), the plane of the sections is indicated by brackets in panel a. ( $f-j)$ Mesothoracic lineage 7 ( $f$ ) Maximum Z projection of the VNS showing the full projection of the metathoracic lineage 7 (green). (b-c) Transverse sections through lineage 7 shown in ( $f$ ), the plane of the sections is indicated by brackets in panel $f$. Dotted lines outline neuropil regions relevant to the projections (sn - primary mechanosensory neuropil, mVAC, ovoid, tectulum and Abdominal neuromeres (Abd). Key tracts and details of the projections are also indicated. Scale $=100 \mu \mathrm{m}$.

Figure 9 MARCM clones for Lineage 8. (a-c) Prothoracic lineage 8 (a) Maximum Z projection of the VNS showing the full projection of the prothoracic lineage 8 (green). (b-c) Transverse sections through lineage 8 shown in (a), the plane of the sections is indicated by brackets in panel a. (d-h) Mesothoracic lineage 8 (g) Maximum Z projection of the VNS showing the full projection of the mesothoracic lineage 8 (green). (h-k) Transverse sections through lineage 6 shown in (d), The plane of the sections is indicated by brackets in panels $\mathrm{d}$. (i-m)

Metathoracic lineage 8 (i) Maximum Z projection of the VNS showing the full projection of the metathoracic lineage 8 (green). ( $j-m$ ) Transverse sections through lineage 8 shown in (i), The plane of the sections is indicated by brackets in panels i. Dotted lines outline neuropil regions relevant to the projections (sn - primary mechanosensory neuropil, ovoid, tectulum and abdominal neuromeres. Key tracts and details of the projections are also indicated. The sample in panel a is incomplete, the posterior elements of the lineage overlap with another clone in $\mathrm{T} 2$, it is not possible to fully differentiate the two projections and posterior elements of lineage 8 in panel a are uncertain. Scale $=100 \mu \mathrm{m}$.

Figure 10 MARCM clones for Lineage 12. (a-d) Prothoracic lineage 12 (a) Maximum Z projection of the VNS showing the full projection of the prothoracic lineage 12 (green). (b-d) Transverse sections through lineage 12 shown in (a), the plane of the sections is indicated by brackets in panel a. (e-h) Mesothoracic lineage 12 (e) Maximum Z projection of the VNS showing the full projection of the mesothoracic lineage 12 (green). (f-h) Transverse sections 
through lineage 6 shown in (e), The plane of the sections is indicated by brackets in panels e. (i-I) Metathoracic lineage 12 (i) Maximum Z projection of the VNS showing the full projection of the metathoracic lineage 12 (green). ( $j-1)$ Transverse sections through lineage 12 shown in (i). The plane of the sections is indicated by brackets in panel i. Dotted lines outline neuropil regions relevant to the projections (sn - primary mechanosensory neuropil, ovoid, tectulum and abdominal neuromeres. Key tracts and details of the projections are also indicated. Scale $=100 \mu \mathrm{m}$.

Figure 11 MARCM clones for Lineages 16 and 17. (a-e) Lineage 16 (a) Maximum Z projection of the VNS showing the full projection of lineage 16 in all three thoracic neuromeres (Pro Green, Meso Cyan and Meta Green). (b-e) Transverse sections through lineage $16 \mathrm{~s}$ shown in (a), the plane of the sections is indicated by brackets in panel a. (f-h) Lineage 17 (f) Maximum Z projection showing the full projection of lineage 17 in the mesothoracic neuromere (Green). (g-h) Transverse sections through lineage 17 shown in (f), the plane of the sections is indicated by brackets in panel $f$. Dotted lines outline neuropil regions relevant to the projections (sn - primary mechanosensory neuropil, ovoid, tectulum and abdominal neuromeres. Key tracts and details of the projections are also indicated. The metathoracic example in panel $a$ is incomplete and shown as the right-hand lineage for clarity. The anterior and contralateral elements of the lineage overlap with other clones and it is not possible to fully differentiate the two projections and some elements of the metathoracic lineage 16 are uncertain. Scale $=100 \mu \mathrm{m}$.

Figure 12 MARCM clones for Lineage 19. (a) Maximum Z projection of the VNS showing the full projection of lineage 19 in the pro and metathoracic neuromeres (Green). (b-e) Transverse sections through lineage 19s shown in (a), the plane of the sections is indicated by brackets in panel a. (f) Maximum Z projection of the VNS showing the full projection of lineage 19 in the mesothoracic neuromere (Green). (g-k) Transverse sections through lineage 19 shown in (f), the plane of the sections is indicated by brackets in panel $f$. Dotted lines outline neuropil regions relevant to the projections (sn - primary mechanosensory neuropil, ovoid, tectulum and abdominal neuromeres. Key tracts and details of the projections are also indicated. Scale $=100 \mu \mathrm{m}$.

Figure 13 MARCM clones for Lineages 20/22 and 21. (a-e) Lineage 20/22 (a) Maximum Z projection of the entire VNS showing the full projection of lineage 20/22 in all three thoracic neuromeres (T1 green, T2 Cyan and T3 magenta). (b-e) Transverse sections through lineages shown in (a). The plane of the sections is indicated by brackets in panel a. (f-k) Lineage 21 (f) Maximum Z projection of the entire VNS showing the full projection of lineage 21in all three thoracic neuromeres (T1 green, T2 Cyan and T3 magenta). (g-k) Transverse sections through lineages shown in (f). The plane of the sections is indicated by brackets in panel $f$. Dotted lines outline neuropil regions relevant to the projections (sn - primary mechanosensory neuropil, ovoid, tectulum and abdominal neuromeres. Key tracts and details of the projections are also indicated. Scale $=100 \mu \mathrm{m}$.

Figure 14 Summary of the functional roles of each hemilineage in a thoracic hemineuromere based on the anatomical organization in the VNS. Schematic presentation of a transverse section through the mesothoracic neuromere summarizing the key findings. Illustrating the approximate distribution of the major arborizations of the mesothoracic 
hemilineages in relation to the major neuropil features and tracts. The hemilineages are organized according to functions inferred from their morphology. Note that where the fine processes of two hemilineages overlap we are unable to fully extract the specific details of each hemilineage. 


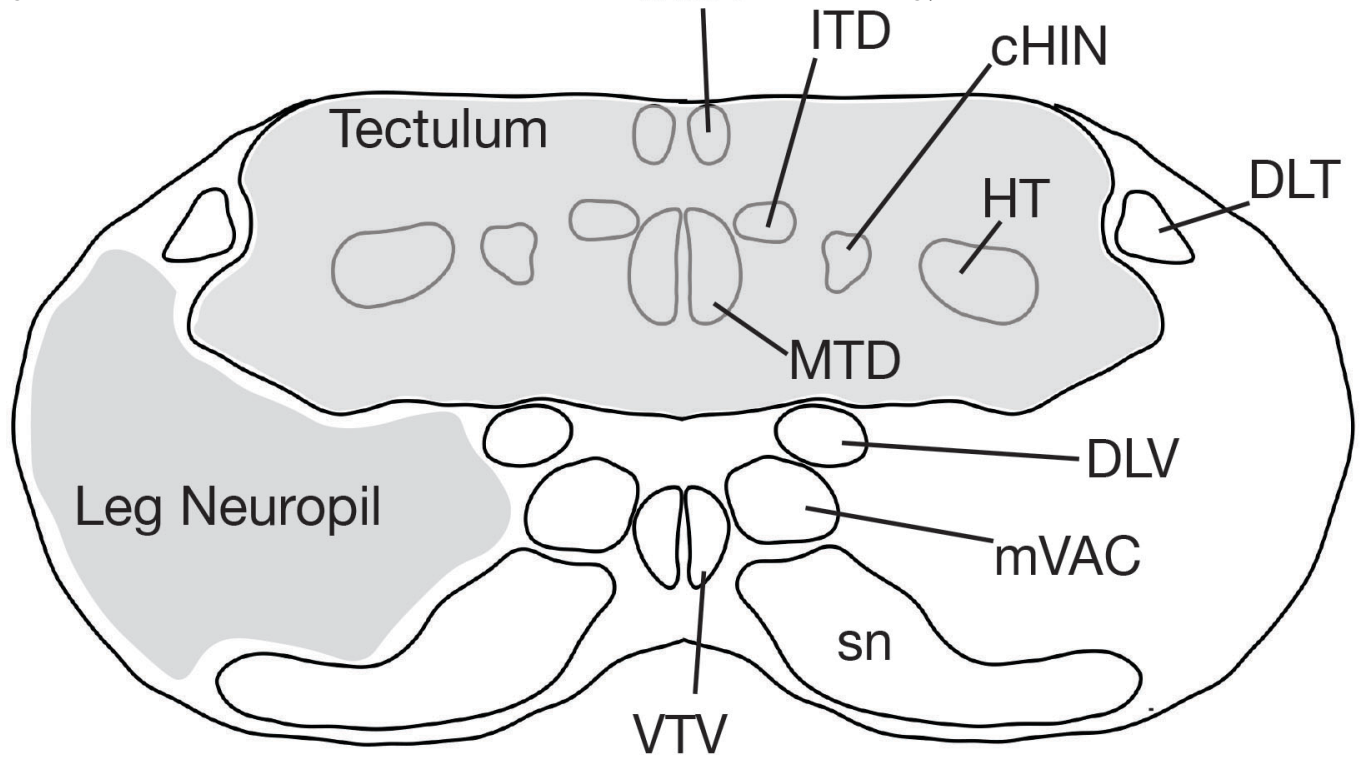

cHIN

DLT

DLV

HT

ITD

MTD

MDT

mVAC

sn

VTV
Contralateral haltere interneurons

Dorsal lateral tract of dorsal fasciculus Dorsal lateral tract of ventral fasciculus Haltere tract Intermediate tract of dorsal fasciculus Median tract of dorsal cervical fasciculus Median dorsal tract Medial ventral association center Leg primany wheepanosensory neuropil Ventral median tract of ventral fasciculus 

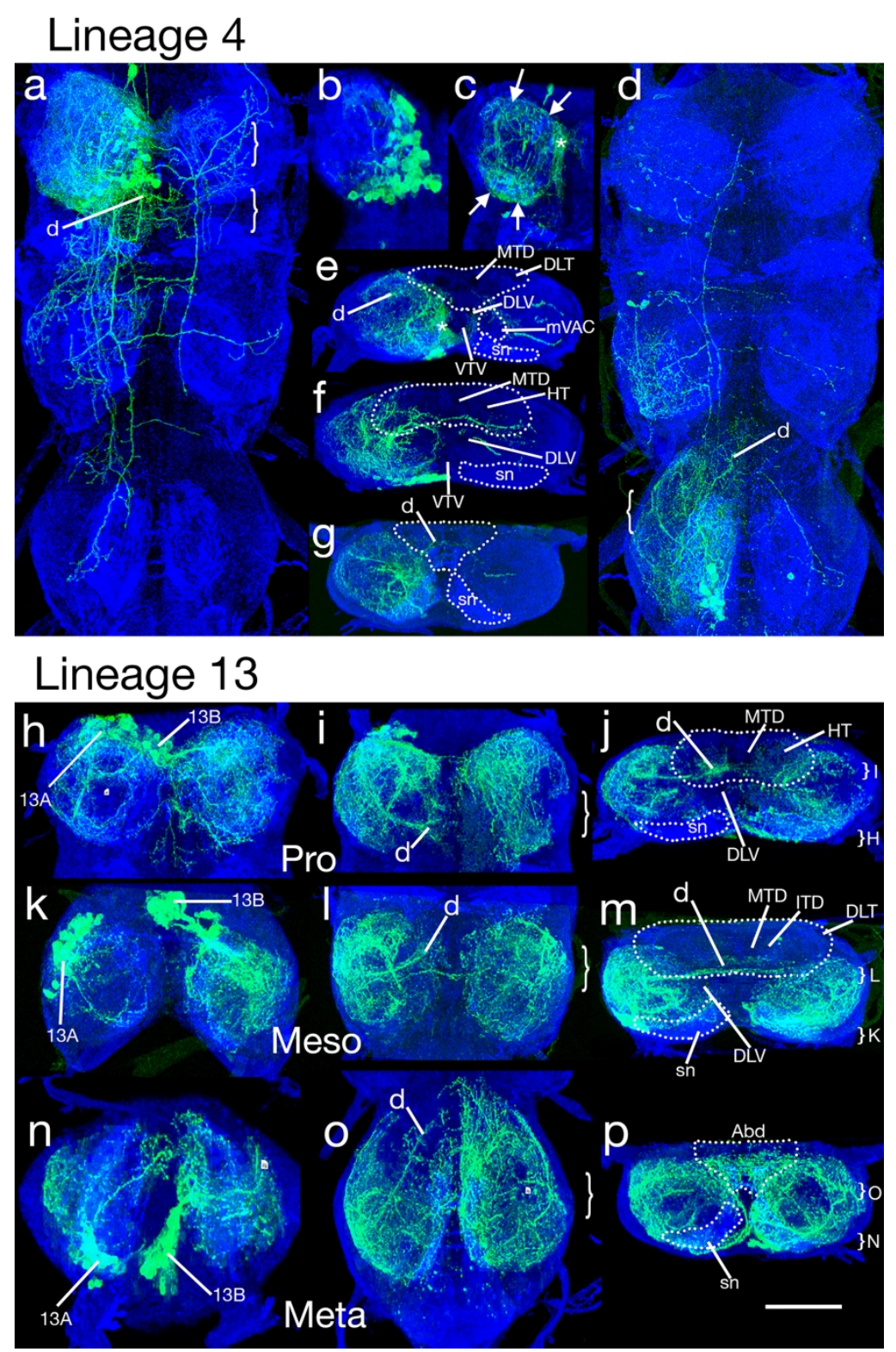

Figure 2 MARCM clones for Lineages 4 and 13. (a-g) Lineage 4 (a) Maximum Z projection of the entire VNS showing the full projection of the prothoracic lineage 4 (green). (b-c) composite of 10 horizontal $Z$ stacks though the ipsilateral prothoracic neuromere selected to reveal details of the lineage. (d) Maximum $Z$ projection of the entire VNS showing the full projection of the metathoracic lineage 4 (green). (e-f) Transverse sections through the specimen shown in (a) the plane of the sections is indicated by the brackets in (a). (g) Transverse section through the specimen shown in (d), the plane of the section is indicated by the bracket in panel D. (h-p) Lineage $13(\mathrm{~h}-\mathrm{i})$ Prothorax - $(\mathrm{h}-\mathrm{i})$ composite of 10 horizontal $\mathrm{Z}$ stacks taken at different dorsal-ventral planes though the prothoracic neuromere selected to reveal details of the lineage, the plane of the sections is indicated by the brackets in $(j)$. (j) Transverse section through the specimen in $(\mathrm{h}-\mathrm{i})$ the plane of the section is indicated by the brackets in (i). (k-m) Mesothorax - $(\mathrm{k}-\mathrm{I})$ composite of 10 horizontal $\mathrm{Z}$ stacks taken at different dorsal-ventral planes though the mesothoracic neuromere selected to reveal details of the lineage, the plane of the sections is indicated by the brackets in $(\mathrm{m})$. ( $\mathrm{I}$ ) Transverse section through the specimen in $(k-m)$ the plane of the section is indicated by the brackets in $(I)$. (n-p) Metathorax - (n-o) composite of 10 horizontal Z stacks taken at different dorsal-ventral planes though the 
metathoracic neuromere selected to reveal details of the lineage, the plane of the sections is indicated by the brackets in (o). (o) Transverse section through the specimen in $(n-0)$ the plane of the section is indicated by the brackets in (o). Dotted lines outline neuropil regions relevant to the projections (sn primary mechanosensory neuropil, mVAC, tectulum and Abd = abdominal neuromeres. Key tracts and details of the projections are also indicated. Scale $=100 \mu \mathrm{m}$. 


\section{Lineage 9}

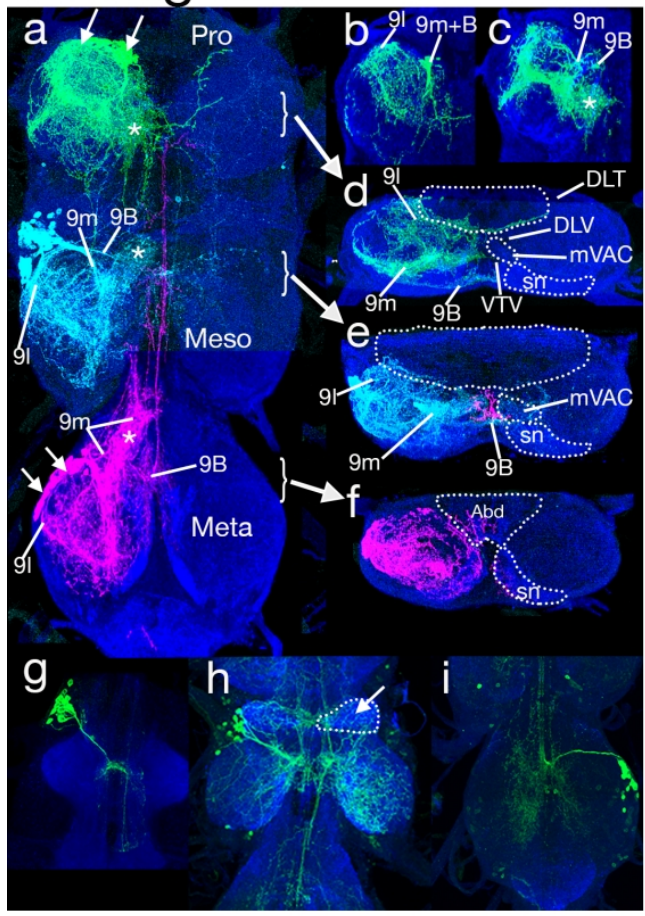

\section{Lineage 23}
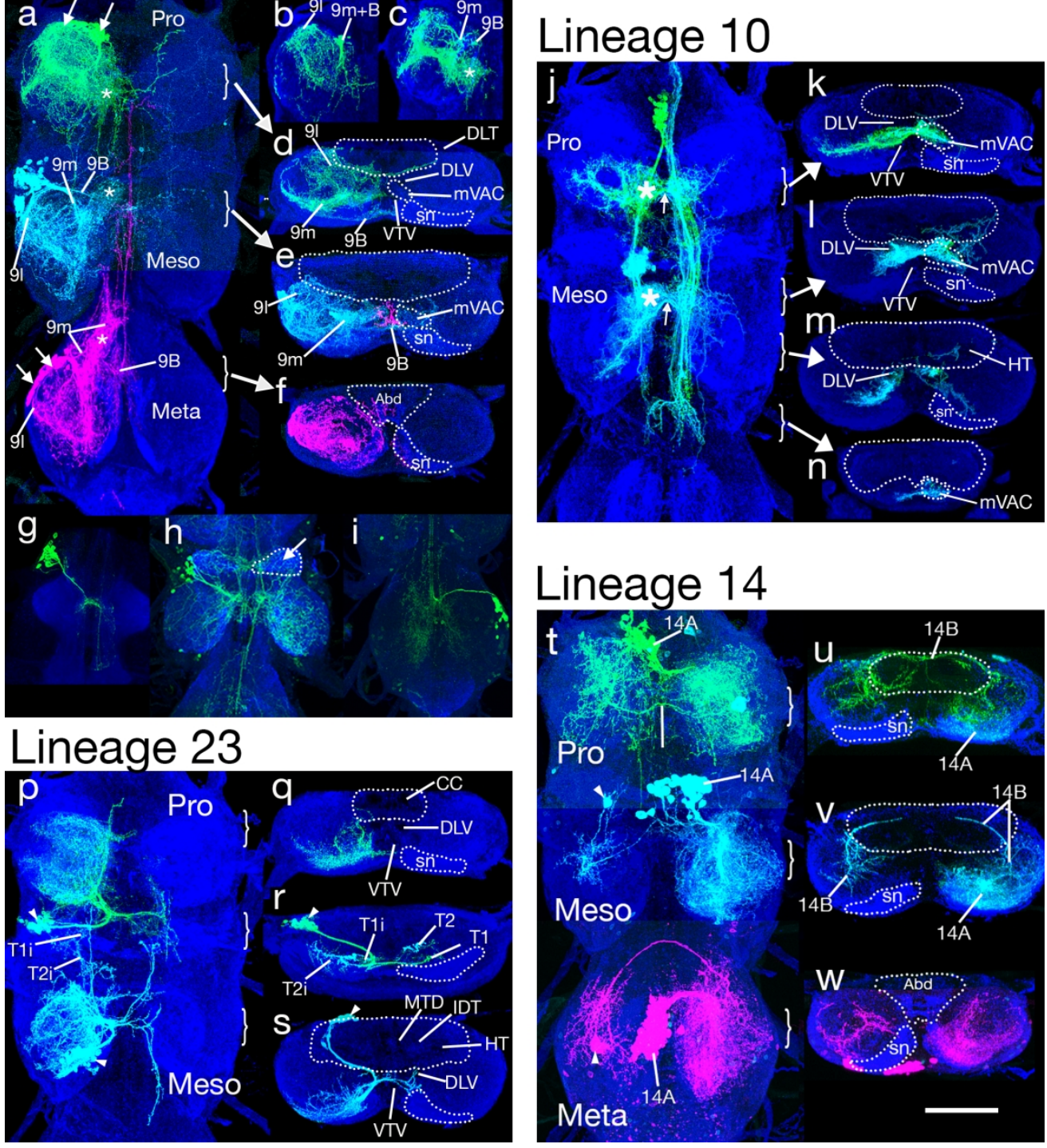

\section{Lineage 14}

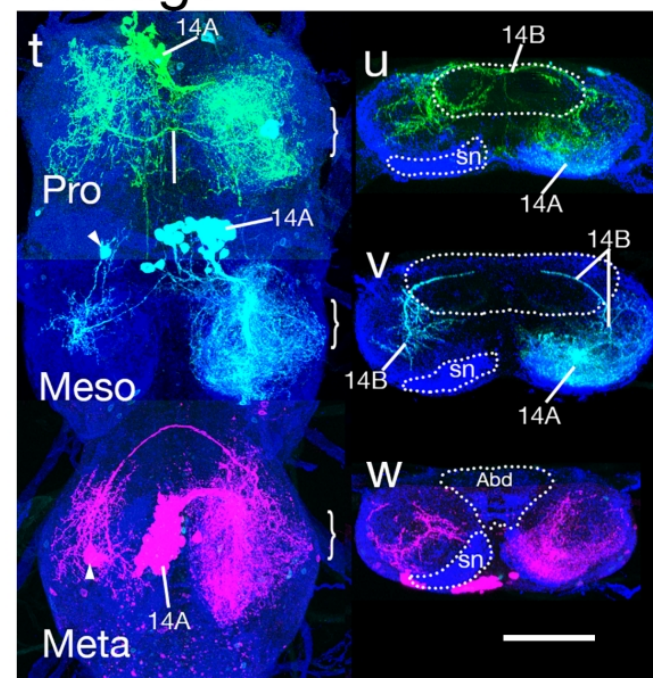

Figure 3 MARCM clones for Lineages 9, 10, 14 and 23. (a-i) Lineage 9 (a) Maximum Z projection of the entire VNS showing the full projection of lineage 9 in all three thoracic neuromeres (T1 green, T2 Cyan and

T3 magenta). (b-c) composite of 10 horizontal $Z$ stacks though the ipsilateral prothoracic neuromere

selected to reveal details of the lineage. (d-f) Transverse sections through lineages shown in (a), (d)

Prothorax, (e) Mesothorax and ( $f$ ) Metathorax. The plane of the sections is indicated by brackets in panel a.

( $g-i)$ Maximum Z projections of OK371 MARCM clones of lineage 9 during pupal stages (green) that

selectively reveals the contralateral component of lineage 9. (g) Prothorax in a pupal VNS at $24 \mathrm{~h}$ after pupation, (h) Mesothorax in a pupal VNS at 48h after pupation, (j) Metathorax in a pupal VNS at $48 \mathrm{~h}$ after pupation. (j-n) Lineage 10 Maximum $Z$ projection of the VNS showing the full projection of lineage 10 in pro and mesothoracic neuromeres (T1 green, T2 Cyan). (k-n) Transverse sections through the preparations shown in ( $j)$, the plane of the sections is indicated by brackets in panel j. ( $p-s$ ) Lineage 23 (p) Maximum Z projection of the VNS showing the full projection of lineage 23 in pro and mesothoracic neuromeres (T1 green, T2 Cyan). (q-s) Transverse sections through the preparations shown in (p), the plane of the sections is indicated by brackets in panel p. (t-w) Lineage $14(\mathrm{t})$ Maximum $\mathrm{Z}$ projection of the VNS showing the full projection of lineage 14 in all three thoracic neuromeres (T1 green, T2 Cyan, T3 Magenta). (u-w) Transverse 
sections through the preparations shown in $(\mathrm{t})$, the plane of the sections is indicated by brackets in panel $t$. Dotted lines outline neuropil regions relevant to the projections (sn - primary mechanosensory neuropil, tectulum and $\mathrm{Abd}=$ abdominal neuromeres. Key tracts and details of the projections are also indicated. Scale $=100 \mu \mathrm{m}$.

$184 \times 212 \mathrm{~mm}(300 \times 300 \mathrm{DPI})$ 


\section{Lineage 2}
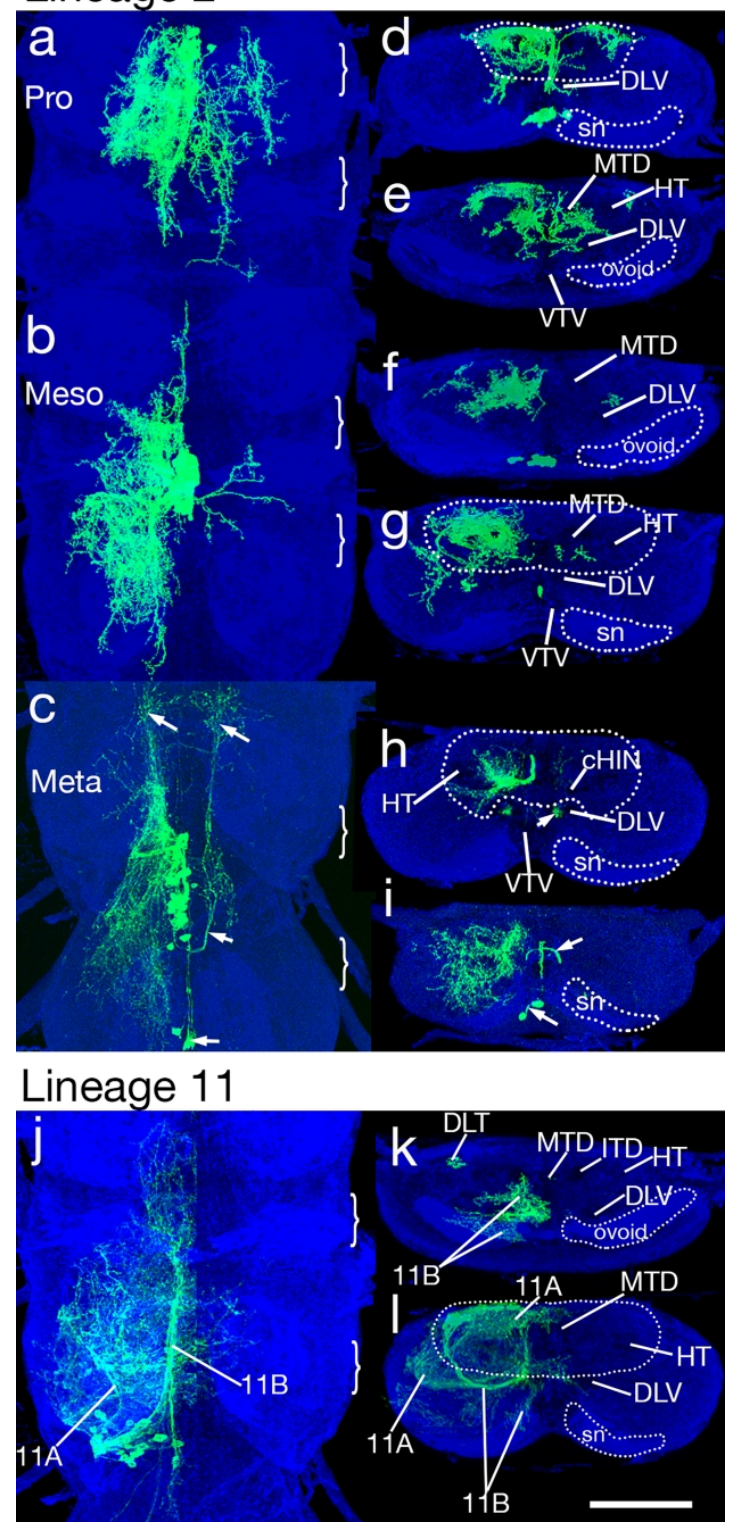

Figure 4 MARCM clones for Lineages 2 and 11. (a-i) Lineage 2 (a-c) Maximum Z projection of the VNS showing the full projection of lineage 2 (green) in all three thoracic neuromeres (a- T1, b- T2 and c- T3). ( $d$ h) Transverse sections through lineages shown in (a-c), The plane of the sections is indicated by brackets in panels a-c. The example in Panels $c, h$ and $i$ includes a single lineage 0 neuron clone (arrows). ( $j-l$ ) Lineage

11 (j) Maximum Z projection of the VNS showing the full projection of lineage 11 (green) in the

mesothoracic neuromere. ( $k-1)$ Transverse sections through lineage 11 shown in $(j)$, The plane of the sections is indicated by brackets in panel $j$. Dotted lines outline neuropil regions relevant to the projections (sn - primary mechanosensory neuropil, ovoid, and tectulum. Key tracts and details of the projections are also indicated. Scale $=100 \mu \mathrm{m}$. 


\section{Lineage 6}
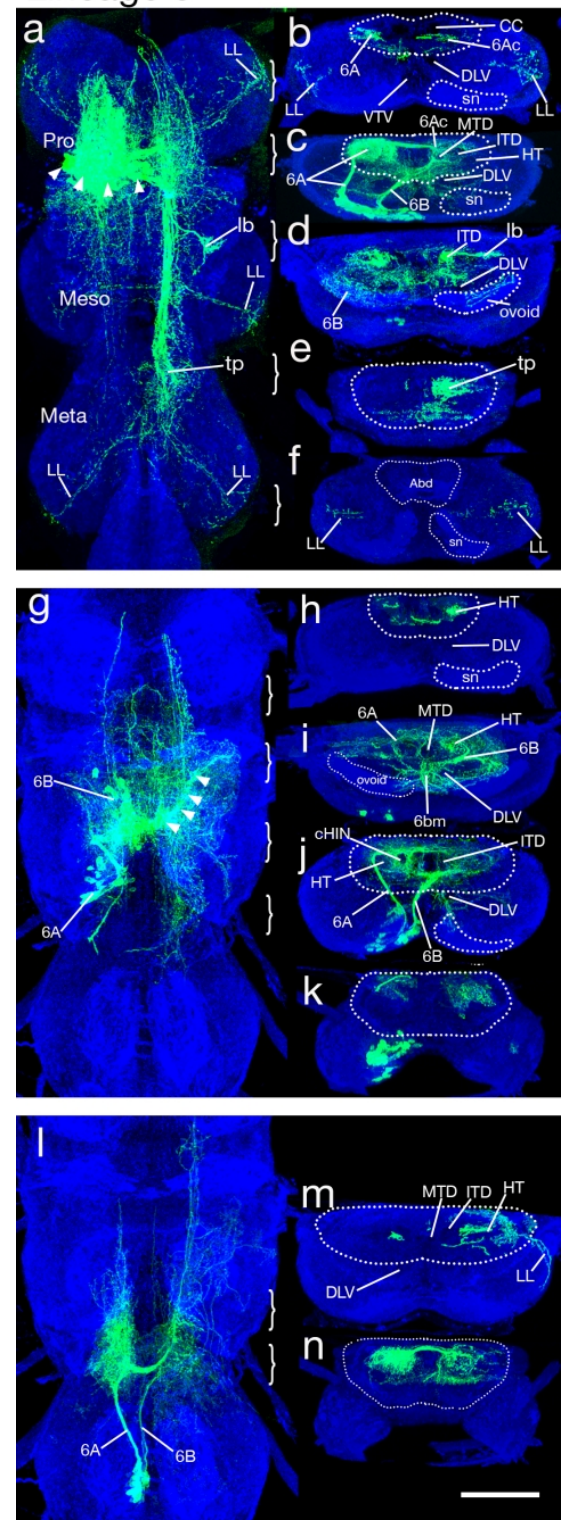

Figure 5 MARCM clones for Lineage 6. (a-f) Prothoracic lineage 6 (a) Maximum Z projection of the VNS showing the full projection of the prothoracic lineage 6 (green). (b-f) Transverse sections through lineage 6 shown in (a), the plane of the sections is indicated by brackets in panel a. (g-k) Mesothoracic lineage 6 ( $g$ ) Maximum Z projection of the VNS showing the full projection of the mesothoracic lineage 6 (green). (h-k) Transverse sections through lineage 6 shown in $(\mathrm{g})$, The plane of the sections is indicated by brackets in panels g. (I-n) Metathoracic lineage 6 (I) Maximum Z projection of the VNS showing the full projection of the metathoracic lineage 6 (green). (m-n) Transverse sections through lineage 6 shown in $(I)$, The plane of the sections is indicated by brackets in panels I. Dotted lines outline neuropil regions relevant to the projections ( $\mathrm{sn}$ - primary mechanosensory neuropil, ovoid, and tectulum. Key tracts and details of the projections are also indicated. Scale $=100 \mu \mathrm{m}$. 

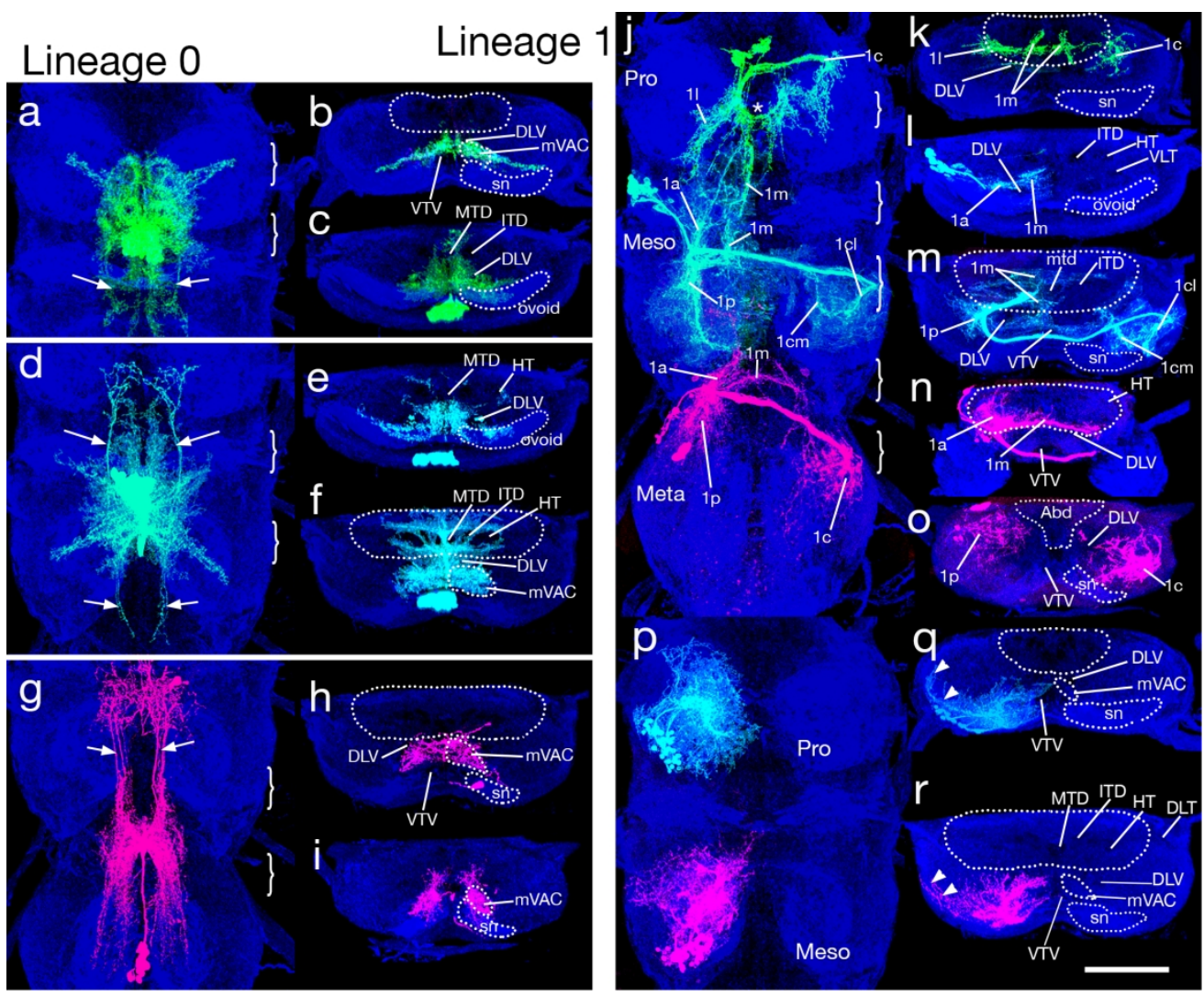

Figure 6 MARCM clones for Lineages 0 and 1. (a-i) Lineage 0 (a-c) Prothoracic lineage 0 . (a) Maximum Z projection of the VNS showing the full projection of the prothoracic lineage 0 (green). (b-c) Transverse sections through lineage 0 shown in (a), the plane of the sections is indicated by brackets in panel a. ( $d-f$ )

Mesothoracic lineage 0 . (d) Maximum Z projection of the VNS showing the full projection of the mesothoracic lineage 0 (green). (e-f) Transverse sections through lineage 0 shown in (d), the plane of the sections is indicated by brackets in panel D. (g-i) Metathoracic lineage 0. (g) Maximum Z projection of the VNS showing the full projection of the metathoracic lineage 0 (green). (h-i) Transverse sections through lineage 0 shown in $(g)$, the plane of the sections is indicated by brackets in panel $g$. $(j-i)$ Lineage $1 A(j)$

Maximum $Z$ projection of the entire VNS showing the full projection of lineage $1 \mathrm{~A}$ in all three thoracic neuromeres (T1 green, T2 Cyan and T3 magenta). (k-r) Transverse sections through lineages shown in (j). The plane of the sections is indicated by brackets in panel j. (p-r) Lineage 1B (p) Maximum Z projection of the VNS showing the full projection of the meso and metathoracic lineage 1Bs (T2 Cyan and T3 magenta).

$(q-r)$ Transverse sections through lineages in ( $p)$, the plane of the sections is indicated by brackets in panelp. Dotted lines outline neuropil regions relevant to the projections (sn - primary mechanosensory neuropil, mVAC, ovoid, tectulum and Abdominal neuromeres (Abd). Key tracts and details of the projections are also indicated. Scale $=100 \mu \mathrm{m}$.

$183 \times 150 \mathrm{~mm}(300 \times 300 \mathrm{DPI})$ 

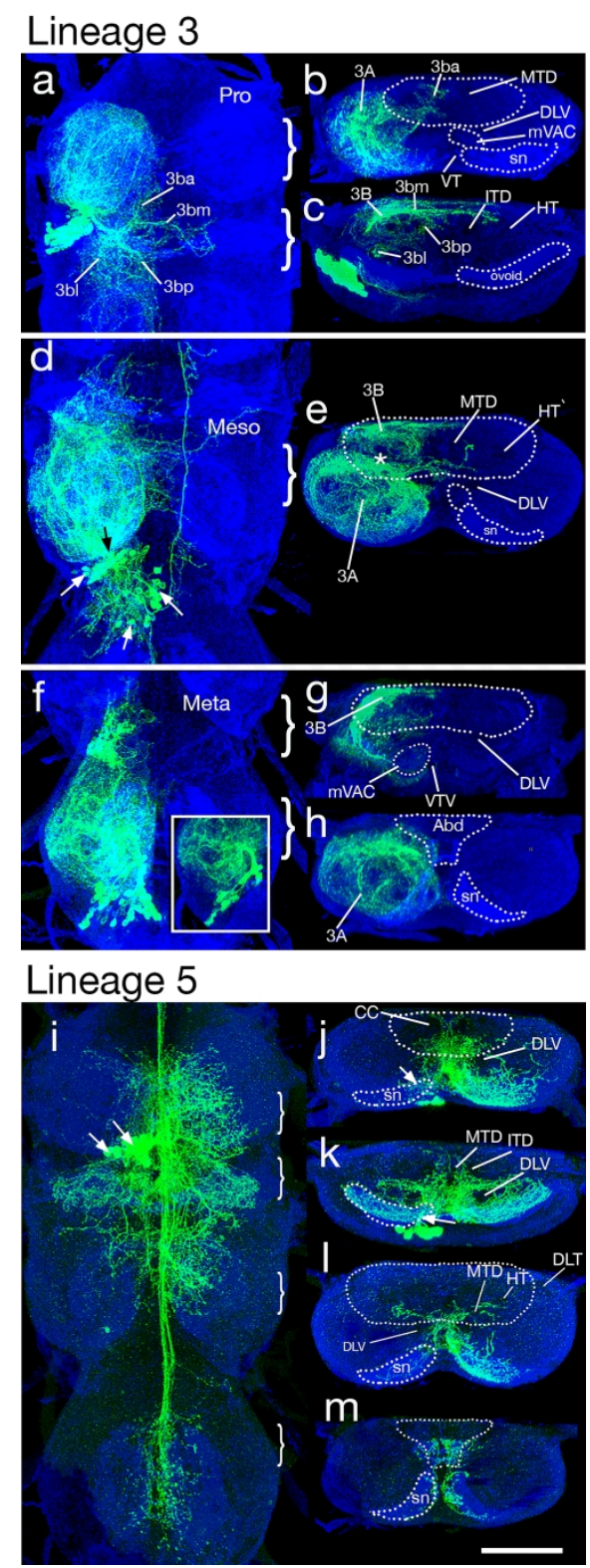

Figure 7 MARCM clones for Lineages 3 and 5. (a-h) Lineage 3 (a-c) Prothoracic lineage 3. (a) Maximum Z projection of the VNS showing the full projection of the prothoracic lineage 3 (green). (b-c) Transverse sections through lineage 3 shown in (a), the plane of the sections is indicated by brackets in panel a. (d-e) Mesothoracic lineage 3. (d) Maximum Z projection of the VNS showing the full projection of the mesothoracic lineage 3 (green). (e) Transverse sections through lineage 3 shown in (d), the plane of the sections is indicated by brackets in panel $d$. ( $f-h)$ Metathoracic lineage 3. (f) Maximum $Z$ projection of the VNS showing the full projection of the metathoracic lineage 3 (green). ( $g-h)$ Transverse sections through lineage 3 shown in (f), the plane of the sections is indicated by brackets in panel $f$. The inset in (f) is a composite of 10 horizontal $Z$ stacks though the ipsilateral metathoracic neuromere selected to reveal the characteristic shape of the primary projection. (i-m) Lineage 5 (I) Maximum Z projection of the VNS showing the full projection of the prothoracic lineage 5 (green). (j-m) Transverse sections through lineage 5 shown in (i), the plane of the sections is indicated by brackets in panel i. Dotted lines outline neuropil regions relevant to the projections (sn - primary mechanosensory neuropil, mVAC, ovoid, tectulum and Abdominal neuromeres $(\mathrm{Abd})$. Key tracts and details of the projections are also indicated. Scale $=100 \mu \mathrm{m}$. 


\section{Lineage 7}
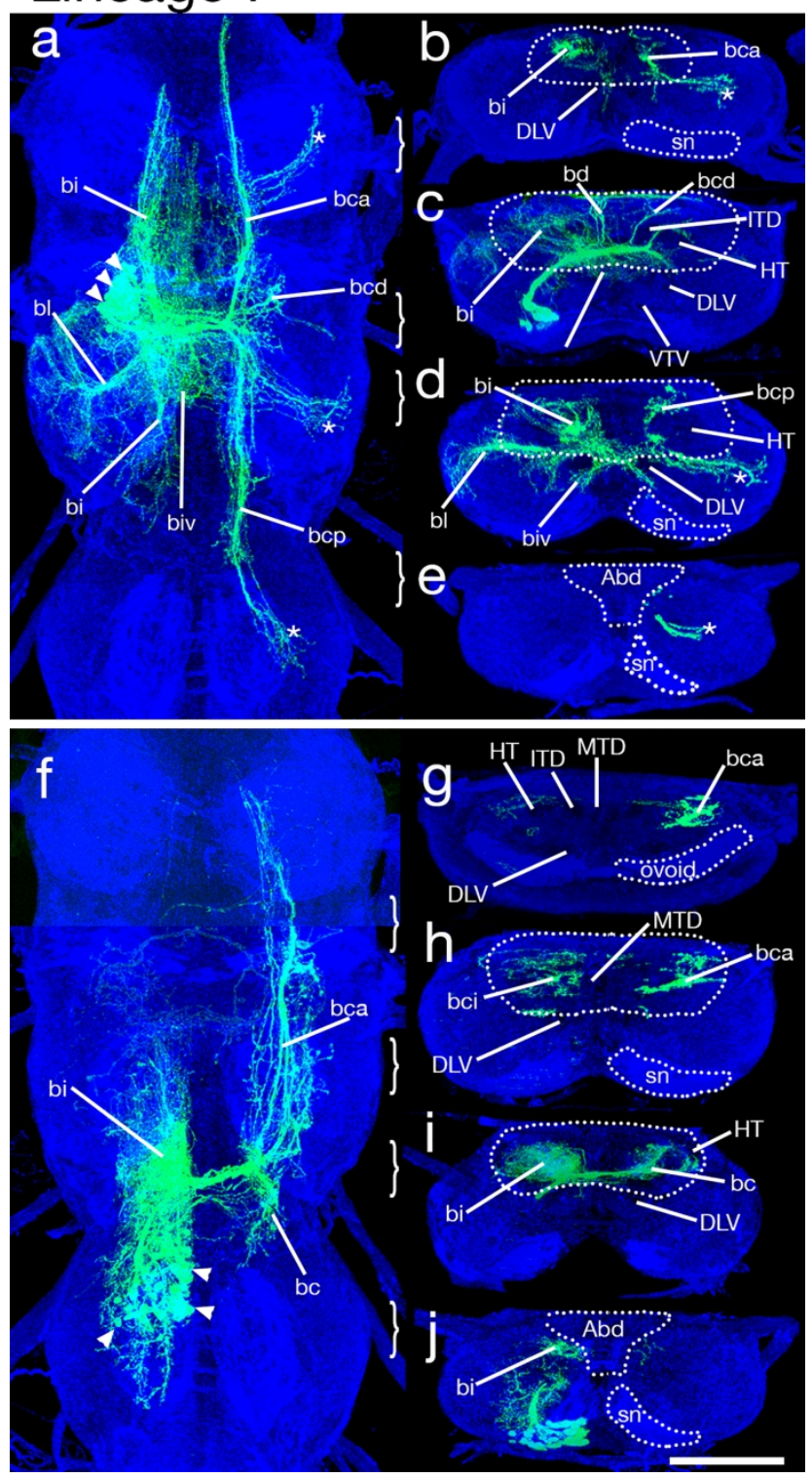

Figure 8 MARCM clones for Lineage 7. (a-e) Mesothoracic lineage 7 (a) Maximum Z projection of the VNS showing the full projection of the mesothoracic lineage 7 (green). (b-c) Transverse sections through lineage 7 shown in (a), the plane of the sections is indicated by brackets in panel $a$. ( $f-j$ ) Mesothoracic lineage 7 ( $f$ ) Maximum Z projection of the VNS showing the full projection of the metathoracic lineage 7 (green). (b-c) Transverse sections through lineage 7 shown in $(f)$, the plane of the sections is indicated by brackets in panel $f$. Dotted lines outline neuropil regions relevant to the projections (sn - primary mechanosensory neuropil, mVAC, ovoid, tectulum and Abdominal neuromeres (Abd). Key tracts and details of the projections are also indicated. Scale $=100 \mu \mathrm{m}$. 


\section{Lineage 8}
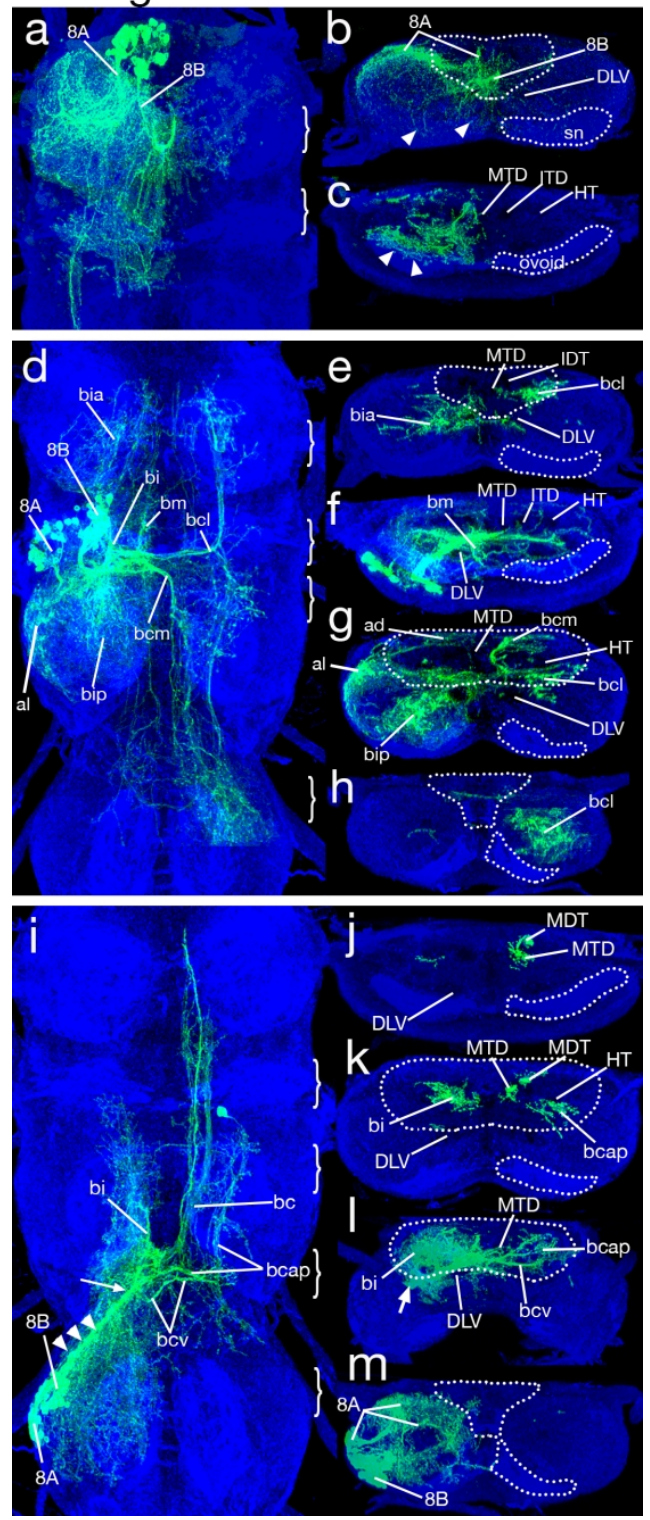

Figure 9 MARCM clones for Lineage 8. (a-c) Prothoracic lineage 8 (a) Maximum Z projection of the VNS showing the full projection of the prothoracic lineage 8 (green). (b-c) Transverse sections through lineage 8 shown in (a), the plane of the sections is indicated by brackets in panel a. (d-h) Mesothoracic lineage 8 ( $g$ ) Maximum Z projection of the VNS showing the full projection of the mesothoracic lineage 8 (green). (h-k) Transverse sections through lineage 6 shown in (d), The plane of the sections is indicated by brackets in panels $\mathrm{d}$. ( $\mathrm{i}-\mathrm{m}$ ) Metathoracic lineage 8 (i) Maximum $\mathrm{Z}$ projection of the VNS showing the full projection of the metathoracic lineage 8 (green). (j-m) Transverse sections through lineage 8 shown in (i), The plane of the sections is indicated by brackets in panels i. Dotted lines outline neuropil regions relevant to the projections (sn - primary mechanosensory neuropil, ovoid, tectulum and abdominal neuromeres. Key tracts and details of the projections are also indicated. The sample in panel a is incomplete, the posterior elements of the lineage overlap with another clone in T2, it is not possible to fully differentiate the two projections and posterior elements of lineage 8 in panel a are uncertain. Scale $=100 \mu m$. 


\section{Lineage 12}
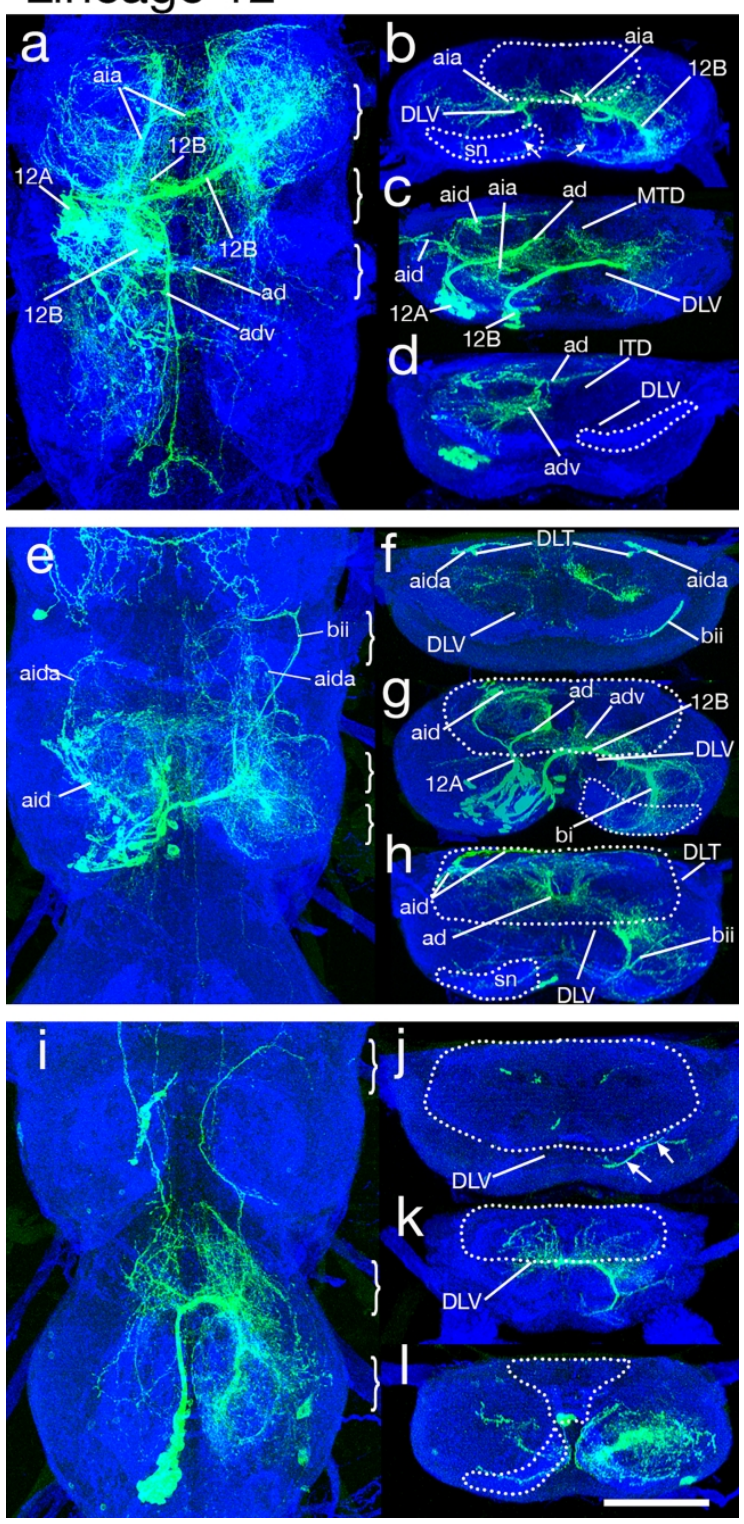

Figure 10 MARCM clones for Lineage 12. (a-d) Prothoracic lineage 12 (a) Maximum Z projection of the VNS showing the full projection of the prothoracic lineage 12 (green). (b-d) Transverse sections through lineage 12 shown in (a), the plane of the sections is indicated by brackets in panel a. (e-h) Mesothoracic lineage 12 (e) Maximum $Z$ projection of the VNS showing the full projection of the mesothoracic lineage 12 (green). ( $f$ h) Transverse sections through lineage 6 shown in (e), The plane of the sections is indicated by brackets in panels e. (i-I) Metathoracic lineage 12 (i) Maximum Z projection of the VNS showing the full projection of the metathoracic lineage 12 (green). ( $j-1)$ Transverse sections through lineage 12 shown in (i). The plane of the sections is indicated by brackets in panel $\mathrm{i}$. Dotted lines outline neuropil regions relevant to the projections (sn - primary mechanosensory neuropil, ovoid, tectulum and abdominal neuromeres. Key tracts and details of the projections are also indicated. Scale $=100 \mu \mathrm{m}$. 


\section{Lineage 16}

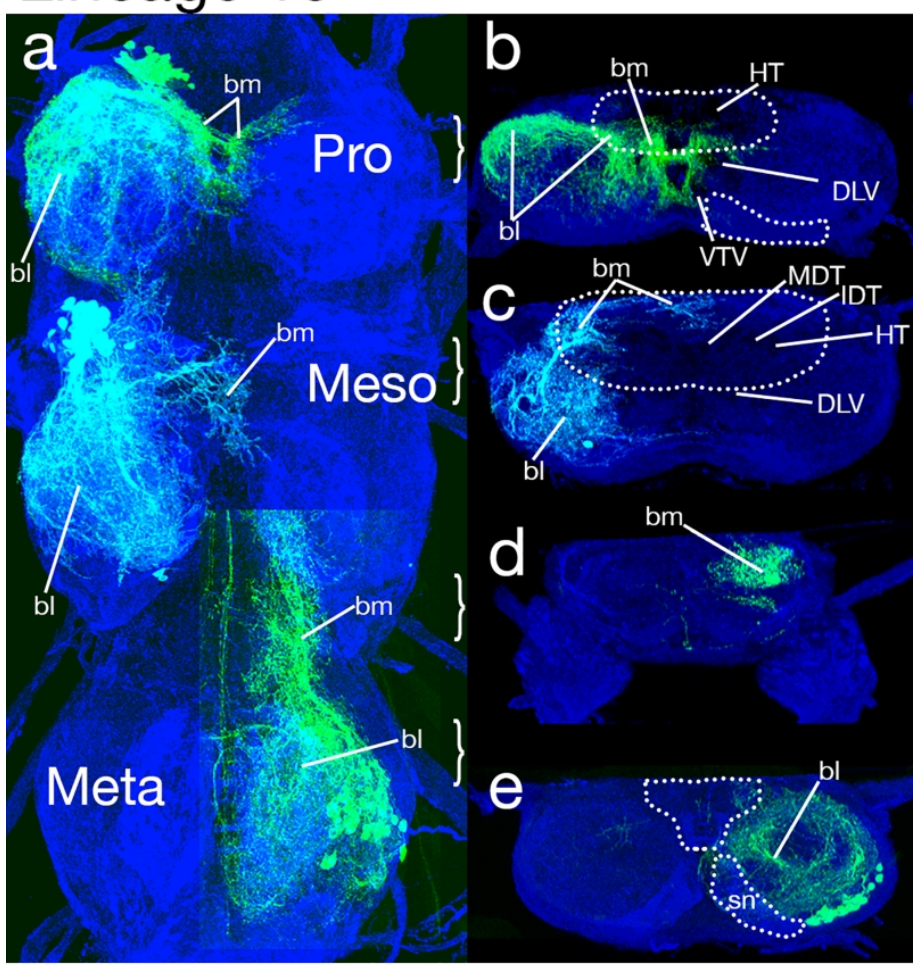

\section{Lineage 17}

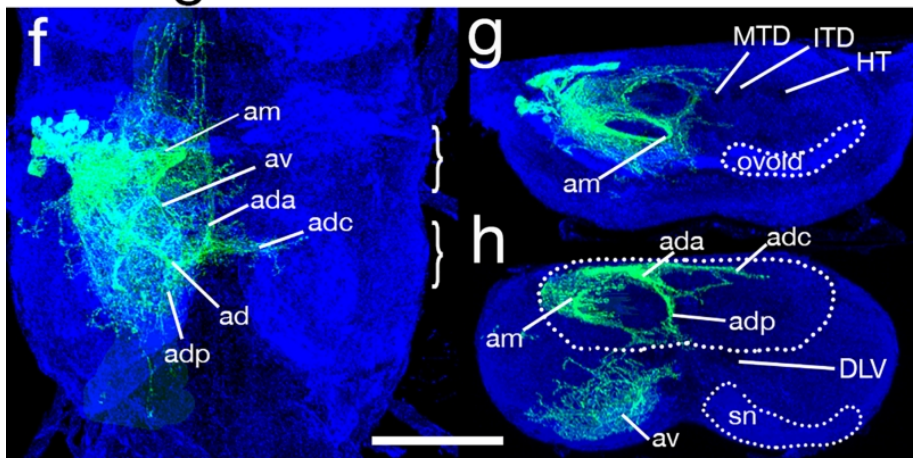

Figure 11 MARCM clones for Lineages 16 and 17. (a-e) Lineage 16 (a) Maximum Z projection of the VNS showing the full projection of lineage 16 in all three thoracic neuromeres (Pro Green, Meso Cyan and Meta Green). (b-e) Transverse sections through lineage $16 \mathrm{~s}$ shown in (a), the plane of the sections is indicated by brackets in panel a. ( $\mathrm{f}-\mathrm{h}$ ) Lineage 17 (f) Maximum $\mathrm{Z}$ projection showing the full projection of lineage 17 in the mesothoracic neuromere (Green). ( $g-h)$ Transverse sections through lineage 17 shown in (f), the plane of the sections is indicated by brackets in panel $\mathrm{f}$. Dotted lines outline neuropil regions relevant to the projections (sn - primary mechanosensory neuropil, ovoid, tectulum and abdominal neuromeres. Key tracts and details of the projections are also indicated. The metathoracic example in panel a is incomplete and shown as the right-hand lineage for clarity. The anterior and contralateral elements of the lineage overlap with other clones and it is not possible to fully differentiate the two projections and some elements of the metathoracic lineage 16 are uncertain. Scale $=100 \mu \mathrm{m}$. 


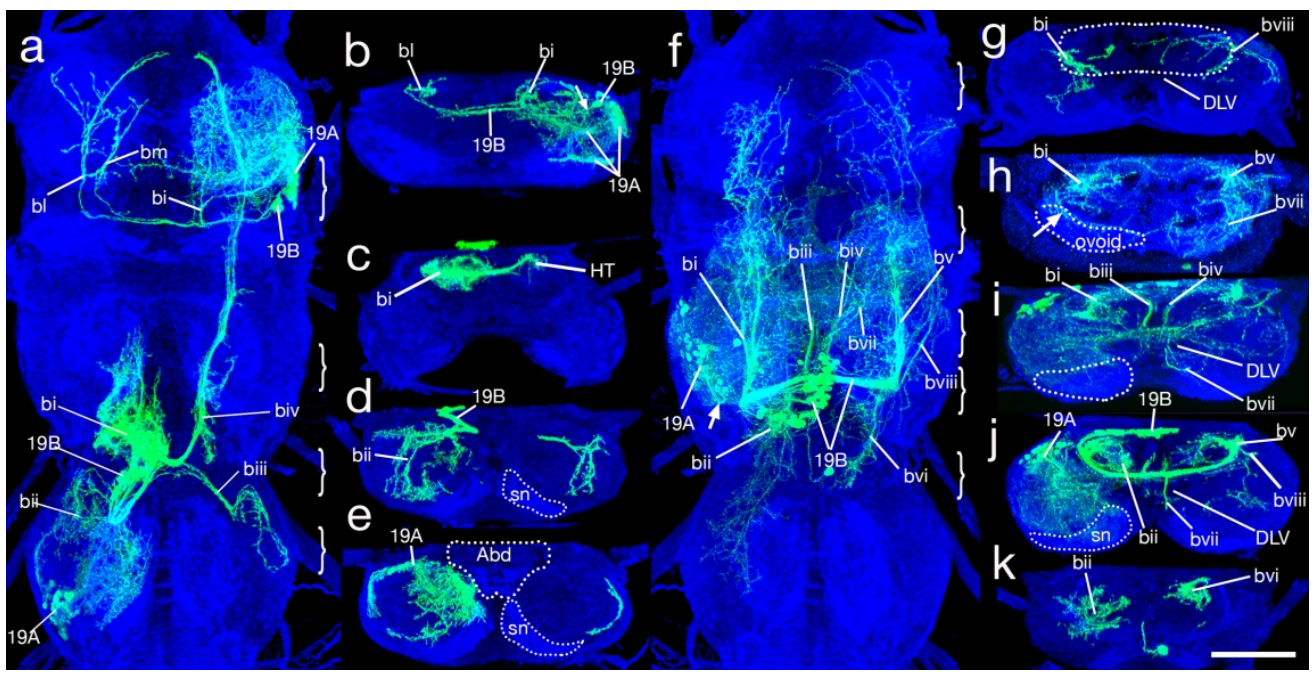

Figure 12 MARCM clones for Lineage 19. (a) Maximum Z projection of the VNS showing the full projection of lineage 19 in the pro and metathoracic neuromeres (Green). (b-e) Transverse sections through lineage 19s shown in (a), the plane of the sections is indicated by brackets in panel a. (f) Maximum $Z$ projection of the VNS showing the full projection of lineage 19 in the mesothoracic neuromere (Green). ( $\mathrm{g}-\mathrm{k}$ ) Transverse sections through lineage 19 shown in (f), the plane of the sections is indicated by brackets in panel f. Dotted lines outline neuropil regions relevant to the projections (sn - primary mechanosensory neuropil, ovoid, tectulum and abdominal neuromeres. Key tracts and details of the projections are also indicated. Scale $=100 \mu \mathrm{m}$. 


\section{Lineage 20/22}

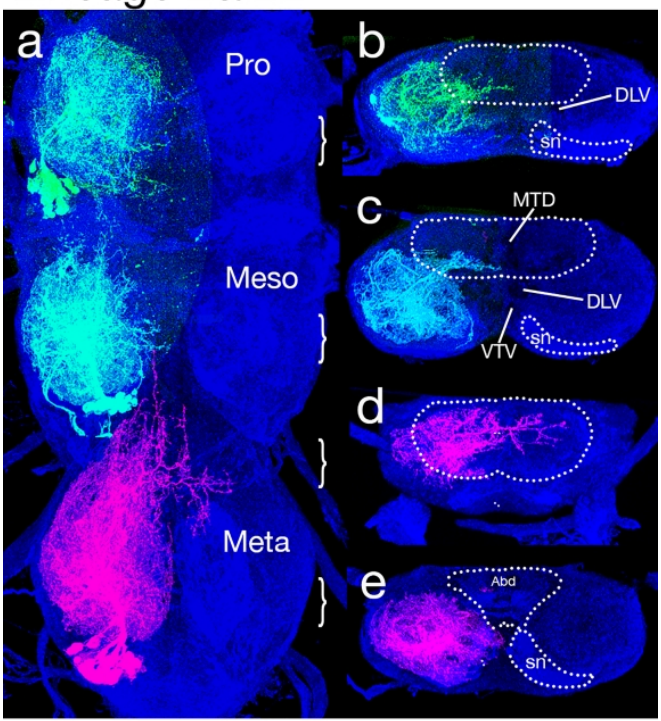

Lineage 21

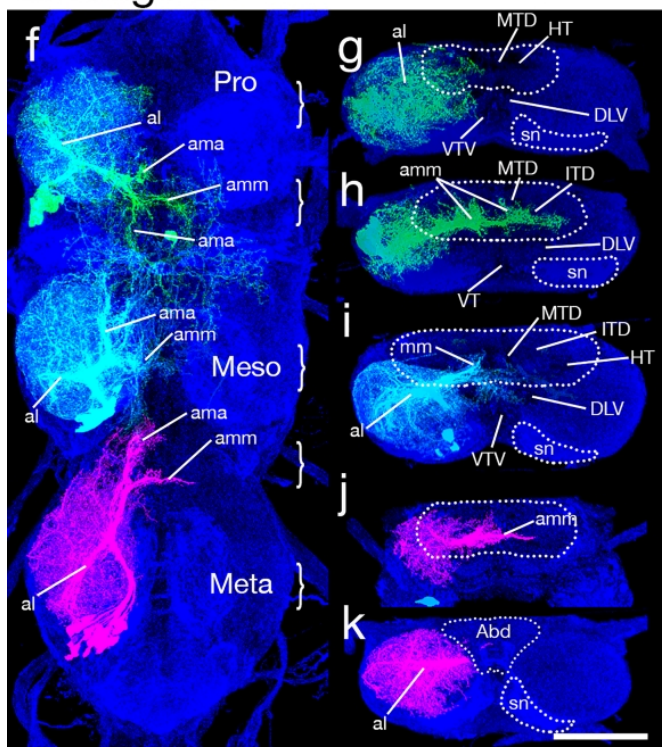

Figure 13 MARCM clones for Lineages 20/22 and 21. (a-e) Lineage 20/22 (a) Maximum Z projection of the entire VNS showing the full projection of lineage 20/22 in all three thoracic neuromeres (T1 green, T2 Cyan and T3 magenta). (b-e) Transverse sections through lineages shown in (a). The plane of the sections is indicated by brackets in panel a. ( $f-k$ ) Lineage 21 (f) Maximum Z projection of the entire VNS showing the full projection of lineage 21in all three thoracic neuromeres (T1 green, T2 Cyan and T3 magenta). (g-k) Transverse sections through lineages shown in (f). The plane of the sections is indicated by brackets in panel f. Dotted lines outline neuropil regions relevant to the projections (sn - primary mechanosensory neuropil, ovoid, tectulum and abdominal neuromeres. Key tracts and details of the projections are also indicated. Scale $=100 \mu \mathrm{m}$. 


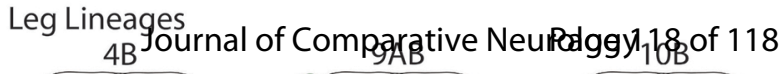
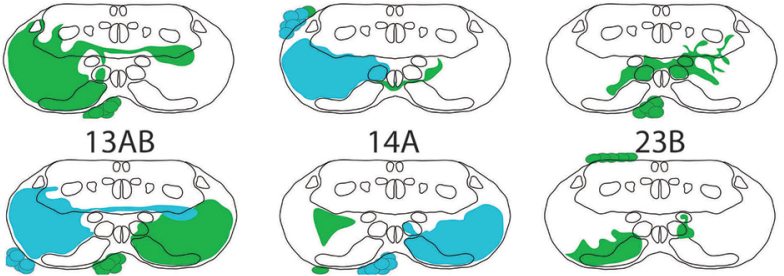

Wing Lineages
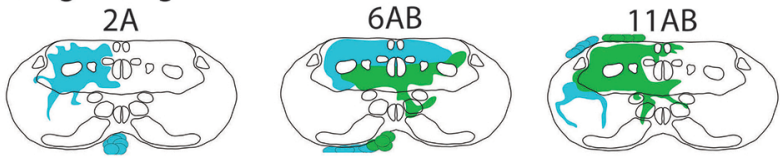

Leg and Wing Lineages

$O A$

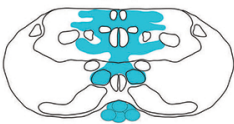

$5 \mathrm{~B}$

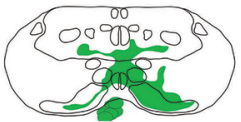

$12 \mathrm{AB}$

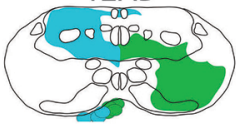

$19 A B$

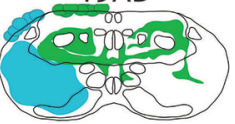

$1 \mathrm{AB}$

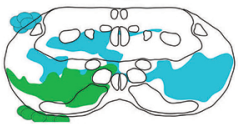

7B

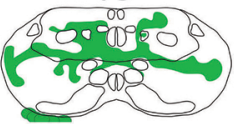

$16 \mathrm{~B}$

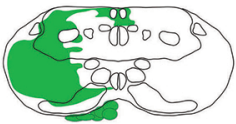

$20 / 22$

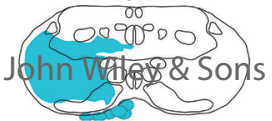

$3 A B$

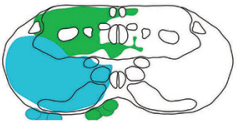

$8 \mathrm{AB}$
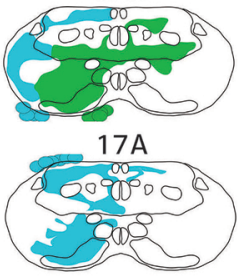

$21 \mathrm{~A}$

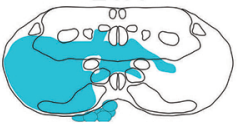




\begin{tabular}{|l|l|l|l|}
\hline Antibody & Immunogen & Dilution & Manufacturer \\
\hline rabbit anti-GFP & GFP & $1: 1000$ & Molecular Probes (Invitrogen) \\
\hline rat anti-N-cadherin & $\begin{array}{l}\text { extracellular domain of } \\
\text { Drosophila N-cadherin }\end{array}$ & $1: 40$ & $\begin{array}{l}\text { Developmental Studies } \\
\text { Hybridoma Bank }\end{array}$ \\
\hline $\begin{array}{l}\text { mouse anti- } \\
\text { neuroglian }\end{array}$ & $\begin{array}{l}\text { nervous system- } \\
\text { specific 180 kD splice } \\
\text { variant of Drosophila } \\
\text { neuroglian }\end{array}$ & $1: 40$ & $\begin{array}{l}\text { Developmental Studies } \\
\text { Hybridoma Bank }\end{array}$ \\
\hline
\end{tabular}

Table 1 Antibodies used in this study 


\begin{tabular}{|c|c|c|c|}
\hline Lineage & Prothorax & Mesothorax & Metathorax \\
\hline $\mathbf{0}$ & 4 & 3 & 2 \\
\hline $\mathbf{1}$ & 7 & 5 & 6 \\
\hline $\mathbf{2}$ & 2 & 8 & 4 \\
\hline $\mathbf{3}$ & 9 & 7 & 4 \\
\hline $\mathbf{4}$ & 2 & 5 & 4 \\
\hline $\mathbf{5}$ & 1 & 0 & 0 \\
\hline $\mathbf{6}$ & 5 & 4 & 5 \\
\hline $\mathbf{7}$ & 0 & 5 & 4 \\
\hline $\mathbf{8}$ & 7 & 4 & 6 \\
\hline $\mathbf{9}$ & 3 & 6 & 6 \\
\hline $\mathbf{1 0}$ & 2 & 1 & 0 \\
\hline $\mathbf{1 1}$ & 0 & 2 & 0 \\
\hline $\mathbf{1 2}$ & 9 & 8 & 10 \\
\hline $\mathbf{1 3}$ & 3 & 2 & 1 \\
\hline $\mathbf{1 4}$ & 10 & 6 & 4 \\
\hline 16 & 5 & 1 & 3 \\
\hline $\mathbf{1 7}$ & 0 & 2 & 0 \\
\hline 18 & 0 & 0 & 0 \\
\hline 19 & 2 & 7 & 4 \\
\hline $\mathbf{2 0 / 2 2}$ & 4 & 6 & 9 \\
\hline $\mathbf{2 1}$ & 4 & 6 & 6 \\
\hline $\mathbf{2 3}$ & 3 & 1 & 0 \\
\hline
\end{tabular}

Table 2. Summary of the number of lineages generated in this study for each neuroblast. 
We have analyzed the adult morphology of most of the postembryonic neurons in the thoracic neuromeres of the Drosophila VNS. The data provide a baseline to understand the functional organization of the VNS and test the developmental rules of neuronal circuit assembly. 


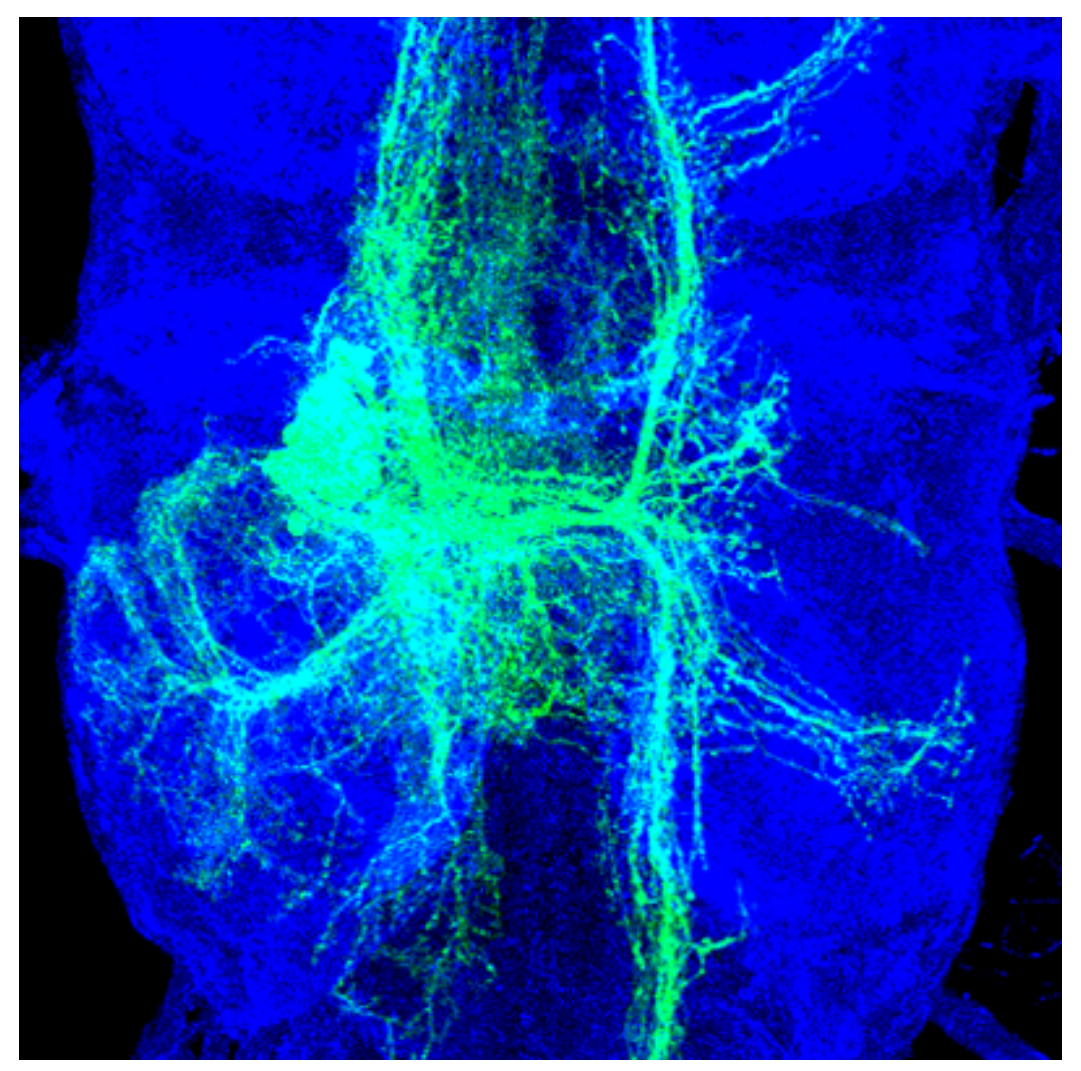

